\title{
IUCrJ
}

Volume 5 (2018)

Supporting information for article:

Thermomechanical effect in molecular crystals: the role of halogenbonding interactions

Sudhir Mittapalli, D. Sravanakumar Perumalla, Jagadeesh Babu Nanubolu and Ashwini Nangia 
Table S1: Hydrogen/halogen bond geometry in crystal structures.

\begin{tabular}{|c|c|c|c|c|c|c|}
\hline & Interaction & D-H/A & $\mathbf{H} \cdot \mathbf{A} / \AA$ & $\mathbf{D} \cdot \mathbf{A} / \AA$ & $\angle \mathbf{D}-\mathbf{H} \cdots \mathbf{A} / \AA$ & Symmetry code \\
\hline \multirow{5}{*}{ A Form I } & $\mathrm{O} 2-\mathrm{H} 2 \mathrm{~A}^{\cdots} \cdots \mathrm{N} 1$ & 0.82 & 1.86 & $2.578(1)$ & 145 & Intramolecular \\
\hline & $\mathrm{N} 2-\mathrm{H} 2 \mathrm{~B} \cdots \mathrm{N} 3$ & 0.86 & 2.17 & $2.9891(2)$ & 156 & $-\mathrm{x}, 1 / 2+\mathrm{y}, 1 / 2-\mathrm{z}$ \\
\hline & $\mathrm{C} 1-\mathrm{H} 1 \cdots \mathrm{Cl} 2$ & 0.93 & 2.81 & $3.722(2)$ & 166 & $\mathrm{x}, 3 / 2-\mathrm{y},-1 / 2+\mathrm{z}$ \\
\hline & $\mathrm{C} 4-\mathrm{Cl} 2 \cdots \mathrm{Cl} 1$ & & & $3.435(6)$ & 155 & Intermolecular \\
\hline & $\mathrm{C} 2-\mathrm{Cl} 1 \cdots \mathrm{Cl} 2$ & & & $3.435(6)$ & 96 & Intermolecular \\
\hline \multirow{7}{*}{ A Form II } & $\mathrm{O} 2-\mathrm{H} 2 \mathrm{~A} \cdots \mathrm{N} 1$ & 0.84 & 1.88 & $2.617(2)$ & 144 & Intramolecular \\
\hline & $\mathrm{N} 2-\mathrm{H} 2 \mathrm{~B} \cdots \mathrm{N} 3$ & 0.86 & 2.24 & $3.083(2)$ & 159 & $-x, 2-y, 1 / 2+z$ \\
\hline & $\mathrm{C} 10-\mathrm{H} 10 \cdots \mathrm{N} 3$ & 0.95 & 2.59 & $3.511(2)$ & 166 & $-\mathrm{x}, 2-\mathrm{y}, 1 / 2+\mathrm{z}$ \\
\hline & $\mathrm{C} 11-\mathrm{H} 11 \cdots \mathrm{O} 1$ & 0.95 & 2.40 & $3.341(2)$ & 174 & $-1 / 2+x, 2-y, z$ \\
\hline & $\mathrm{C} 13-\mathrm{H} 13 \cdots \mathrm{Cl1}$ & 0.95 & 2.92 & $3.676(2)$ & 136 & $\mathrm{x}, 1+\mathrm{y},-1+\mathrm{z}$ \\
\hline & $\mathrm{C} 4-\mathrm{Cl} 2 \cdots \mathrm{Cl} 1$ & & & $3.395(1)$ & 151 & Intermolecular \\
\hline & $\mathrm{C} 2-\mathrm{Cl} 1 \cdots \mathrm{Cl} 2$ & & & $3.395(1)$ & 100 & Intermolecular \\
\hline \multirow{6}{*}{ A Form III } & $\mathrm{O} 2-\mathrm{H} 2 \mathrm{~A} \cdots \mathrm{N} 1$ & 0.82 & 1.88 & $2.591(11)$ & 144 & Intramolecular \\
\hline & $\mathrm{N} 2-\mathrm{H} 2 \mathrm{~B} \cdots \mathrm{N} 3$ & 0.86 & 2.23 & $3.048(11)$ & 159 & $2-x,-1 / 2+y, 1 / 2-z$ \\
\hline & C10-H10 N N3 & 0.93 & 2.56 & $3.466(14)$ & 166 & $2-\mathrm{x},-1 / 2+\mathrm{y}, 1 / 2-\mathrm{z}$ \\
\hline & $\mathrm{C} 11-\mathrm{H} 11 \cdots \mathrm{O} 1$ & 0.93 & 2.47 & $3.391(12)$ & 174 & $\mathrm{x}, 1 / 2-\mathrm{y}, 1 / 2+\mathrm{z}$ \\
\hline & $\mathrm{C} 4-\mathrm{Cl} 2 \cdots \mathrm{Cl} 1$ & & & $3.448(4)$ & 155.5 & Intermolecular \\
\hline & $\mathrm{C} 2-\mathrm{Cl} 1 \cdots \mathrm{Cl} 2$ & & & $3.448(4)$ & 95 & Intermolecular \\
\hline \multirow{9}{*}{ B Form I } & $\mathrm{O} 2-\mathrm{H} 2 \mathrm{~A} \cdots \mathrm{N} 1$ & 0.82 & 1.83 & $2.551(4)$ & 146 & Intramolecular \\
\hline & $\mathrm{N} 2-\mathrm{H} 2 \mathrm{~B} \cdots \mathrm{N} 3$ & 0.86 & 2.13 & $2.920(5)$ & 152 & $-\mathrm{x},-1 / 2+\mathrm{y}, 1 / 2-\mathrm{z}$ \\
\hline & $\mathrm{C} 12-\mathrm{H} 12 \cdots \mathrm{O} 1$ & 0.93 & 2.45 & $3.354(5)$ & 165 & $\mathrm{x}, 1 / 2-\mathrm{y}, 1 / 2+\mathrm{z}$ \\
\hline & $\mathrm{C} 4-\mathrm{Br} 2 \cdots \mathrm{Br} 1$ & & & $3.587(1)$ & 166 & Intermolecular \\
\hline & $\mathrm{C} 2-\mathrm{Br} 1 \cdots \mathrm{Br} 2$ & & & $3.587(1)$ & 94 & Intermolecular \\
\hline & $\mathrm{O} 2-\mathrm{H} 2 \mathrm{~A} \cdots \mathrm{N} 1$ & 0.82 & 1.89 & $2.605(5)$ & 143 & Intramolecular \\
\hline & $\mathrm{N} 2-\mathrm{H} 2 \mathrm{~B} \cdots \mathrm{O} 3$ & 0.80 & 2.15 & $2.933(6)$ & 164 & $\mathrm{X}, \mathrm{y}, \mathrm{z}$ \\
\hline & O4-H4A $\cdots \mathrm{N} 4$ & 0.82 & 1.88 & $2.598(6)$ & 145 & $\mathrm{X}, \mathrm{y}, \mathrm{z}$ \\
\hline & $\mathrm{N} 5-\mathrm{H} 5 \mathrm{~B} \cdots \mathrm{O} 1$ & 0.73 & 2.11 & $2.836(6)$ & 169 & $1-x, 1-y, 1-z$ \\
\hline
\end{tabular}




\begin{tabular}{|c|c|c|c|c|c|c|}
\hline \multirow[t]{3}{*}{ B Form II } & C10-H10 $\cdots$ O3 & 0.93 & 2.51 & $3.417(7)$ & 166 & $\mathrm{X}, \mathrm{y}, \mathrm{z}$ \\
\hline & $\mathrm{C} 23-\mathrm{H} 23 \cdots \mathrm{Br} 3$ & 0.93 & 2.91 & $3.707(6)$ & 145 & $1-x, 1-y,-z$ \\
\hline & $\mathrm{C} 15-\mathrm{Br} 3 \cdots \mathrm{Br} 3$ & & & $3.415(1)$ & 143 & Intermolecular \\
\hline \multirow[t]{5}{*}{ C Form I } & $\mathrm{O} 2-\mathrm{H} 2 \mathrm{~A}^{\cdots} \cdots \mathrm{N} 1$ & 0.82 & 1.85 & $2.565(3)$ & 145 & Intramolecular \\
\hline & $\mathrm{N} 2-\mathrm{H} 2 \mathrm{~B} \cdots \mathrm{N} 3$ & 0.86 & 2.16 & $2.966(3)$ & 156 & $2-\mathrm{x}, 1 / 2+\mathrm{y}, 1 / 2-\mathrm{z}$ \\
\hline & $\mathrm{C} 11-\mathrm{H} 11 \cdots \mathrm{O} 1$ & 0.93 & 2.46 & $3.391(3)$ & 173 & $\mathrm{x}, 1 / 2-\mathrm{y},-1 / 2+\mathrm{z}$ \\
\hline & $\mathrm{C} 4-\mathrm{Br} 1 \cdots \mathrm{Cl1}$ & & & $3.4962(9)$ & 167 & Intermolecular \\
\hline & $\mathrm{C} 2-\mathrm{Cl1} \cdots \mathrm{Br} 1$ & & & $3.4962(9)$ & 98 & Intermolecular \\
\hline \multirow{5}{*}{ C Form II } & $\mathrm{O} 2-\mathrm{H} 2 \mathrm{~A} \cdots \mathrm{N} 1$ & 0.82 & 1.86 & $2.568(4)$ & 145 & Intramolecular \\
\hline & $\mathrm{N} 2-\mathrm{H} 2 \mathrm{~B} \cdots \mathrm{N} 3$ & 0.86 & 2.22 & $3.028(9)$ & 156 & $-\mathrm{x}, 1 / 2+\mathrm{y}, 1 / 2-\mathrm{z}$ \\
\hline & $\mathrm{C} 11-\mathrm{H} 11 \cdots \mathrm{O} 1$ & 0.93 & 2.50 & $3.427(4)$ & 177 & $\mathrm{x}, 3 / 2-\mathrm{y}, 1 / 2+\mathrm{z}$ \\
\hline & $\mathrm{C} 4-\mathrm{Br} 1 \cdots \mathrm{Cl1}$ & & & $3.50(1)$ & 163 & Intermolecular \\
\hline & $\mathrm{C} 2-\mathrm{Cl} 1 \cdots \mathrm{Br} 1$ & & & $3.50(1)$ & 98 & Intermolecular \\
\hline \multirow{4}{*}{ Compound-D } & $\mathrm{O} 2-\mathrm{H} 2 \mathrm{~A}^{\cdots} \cdots \mathrm{N} 1$ & 0.84 & 1.83 & $2.564(2)$ & 145 & Intramolecular \\
\hline & $\mathrm{N} 2-\mathrm{H} 2 \mathrm{~B} \cdots \mathrm{N} 3$ & 0.88 & 2.15 & $2.920(2)$ & 146 & $-\mathrm{x},-1 / 2+\mathrm{y}, 1 / 2-\mathrm{z}$ \\
\hline & $\mathrm{C} 4-\mathrm{I} 2 \cdots \mathrm{I} 1$ & & & $3.8561(5)$ & 170 & Intermolecular \\
\hline & $\mathrm{C} 2-\mathrm{I} 1 \cdots \mathrm{I} 2$ & & & $3.8561(5)$ & 92 & Intermolecular \\
\hline \multirow[t]{2}{*}{ Compound-E } & $\mathrm{O} 2-\mathrm{H} 2 \mathrm{~A}^{\cdots} \cdots \mathrm{N} 1$ & 0.82 & 1.90 & $2.6062(7)$ & 144 & Intramolecular \\
\hline & $\mathrm{N} 2-\mathrm{H} 2 \mathrm{~B} \cdots \mathrm{N} 3$ & 0.86 & 2.19 & $3.0130(8)$ & 162 & $1-\mathrm{x},-1 / 2+\mathrm{y}, 3 / 2-\mathrm{z}$ \\
\hline
\end{tabular}

Table S2: Crystallographic information.

\begin{tabular}{|c|c|c|c|c|c|}
\hline & $\begin{array}{c}\text { Compound-A } \\
\text { Form I }\end{array}$ & $\begin{array}{c}\text { Compound-A } \\
\text { Form II }\end{array}$ & $\begin{array}{c}\text { Compound-A } \\
\text { Form III }\end{array}$ & $\begin{array}{c}\text { Compound-B } \\
\text { Form I }\end{array}$ & $\begin{array}{c}\text { Compound-B } \\
\text { Form II }\end{array}$ \\
\hline Empirical Formula & $\mathrm{C}_{13} \mathrm{H}_{9} \mathrm{Cl}_{2} \mathrm{~N}_{3} \mathrm{O}_{2}$ & $\mathrm{C}_{13} \mathrm{H}_{9} \mathrm{Cl}_{2} \mathrm{~N}_{3} \mathrm{O}_{2}$ & $\mathrm{C}_{13} \mathrm{H}_{9} \mathrm{Cl}_{2} \mathrm{~N}_{3} \mathrm{O}_{2}$ & $\mathrm{C}_{13} \mathrm{H}_{9} \mathrm{Br}_{2} \mathrm{~N}_{3} \mathrm{O}_{2}$ & $\mathrm{C}_{13} \mathrm{H}_{9} \mathrm{Br}_{2} \mathrm{~N}_{3} \mathrm{O}_{2}$ \\
\hline Formula weight & 310.13 & 310.13 & 310.13 & 399.05 & 399.05 \\
\hline Crystal system & Monoclinic & Orthorhombic & Monoclinic & Monoclinic & Triclinic \\
\hline Space group & $P 2_{1} / c$ & $P c a 2_{1}$ & $P 2_{1} / c$ & $P 22_{1} / c$ & $P-1$ \\
\hline $\mathrm{T}(\mathrm{K})$ & 298 & 298 & 338 & 298 & 298 \\
\hline
\end{tabular}




\begin{tabular}{|c|c|c|c|c|c|}
\hline$a(\AA)$ & $8.0683(4)$ & $15.441(3)$ & $8.6916(6)$ & $8.266(2)$ & $9.8084(7)$ \\
\hline$b(\AA)$ & $10.7464(6)$ & $8.3179(16)$ & $10.2641(7)$ & $10.917(2)$ & $10.7751(8)$ \\
\hline$c(\AA)$ & $15.6155(8)$ & $10.6014(18)$ & $15.4965(10)$ & $15.734(3)$ & $13.9516(9)$ \\
\hline$\alpha(\mathrm{deg})$ & 90 & 90 & 90 & 90 & $103.219(3)$ \\
\hline$\beta(\mathrm{deg})$ & $90.890(2)$ & 90 & $90.186(2)$ & $95.82(3)$ & $94.907(3)$ \\
\hline$\gamma(\mathrm{deg})$ & 90 & 90 & 90 & 90 & $91.018(3)$ \\
\hline$V\left(\AA^{3}\right)$ & $1353.78(12)$ & $1361.6(5)$ & $1382.46(16)$ & $1412.7(5)$ & 1429.11(17) \\
\hline$D_{\text {calcd }}\left(\mathrm{g} \mathrm{cm}^{-3}\right)$ & 1.522 & 1.513 & 1.490 & 1.876 & 1.855 \\
\hline$\mu\left(\mathrm{mm}^{-1}\right)$ & 0.483 & 0.480 & 0.473 & 5.742 & 5.676 \\
\hline$\theta$ range & 2.30 to 27.57 & 2.44 to 25.03 & 2.34 to 25.07 & 3.10 to 28.05 & 2.45 to 24.79 \\
\hline $\mathrm{Z} / \mathrm{Z}$ & $4 / 1$ & $4 / 1$ & $4 / 1$ & $4 / 1$ & $4 / 2$ \\
\hline Range $h$ & -10 to 10 & -18 to 18 & -10 to 9 & -10 to 10 & -11 to 11 \\
\hline Range $k$ & -13 to 13 & -9 to 9 & -12 to 12 & -14 to 14 & -12 to 12 \\
\hline Range $l$ & -20 to 20 & -12 to 12 & -18 to 18 & -20 to 20 & -16 to 16 \\
\hline Reflections collected & 35148 & 8992 & 12375 & 18639 & 28553 \\
\hline Total reflections & 3124 & 2379 & 2046 & 3402 & 4894 \\
\hline Observed reflections & 2690 & 2279 & 1677 & 2697 & 4061 \\
\hline$R_{1}[I>2 \sigma(I)]$ & 0.032 & 0.024 & 0.0811 & 0.0470 & 0.0478 \\
\hline$w R 2$ (all) & 0.0916 & 0.0626 & 0.2943 & 0.1028 & 0.1180 \\
\hline Goodness-of-fit & 1.034 & 1.096 & 1.535 & 1.053 & 1.042 \\
\hline X-ray diffractometer & $\begin{array}{c}\text { Bruker D8 } \\
\text { Quest }\end{array}$ & $\begin{array}{c}\text { Bruker D8 } \\
\text { Quest }\end{array}$ & $\begin{array}{c}\text { Bruker D8 } \\
\text { Venture }\end{array}$ & $\begin{array}{c}\text { Bruker D8 } \\
\text { Quest }\end{array}$ & $\begin{array}{c}\text { Bruker D8 } \\
\text { Quest }\end{array}$ \\
\hline CCDC Numbers & 824932 & 1548278 & 1548279 & 1548280 & 1548281 \\
\hline
\end{tabular}




\begin{tabular}{|c|c|c|c|c|}
\hline & $\begin{array}{c}\text { Compound-C } \\
\text { Form I }\end{array}$ & $\begin{array}{c}\text { Compound-C } \\
\text { Form II }\end{array}$ & Compound-D & Compound-E \\
\hline Empirical Formula & $\mathrm{C}_{13} \mathrm{H}_{9} \mathrm{BrClN}_{3} \mathrm{O}_{2}$ & $\mathrm{C}_{13} \mathrm{H}_{9} \mathrm{BrClN}_{3} \mathrm{O}_{2}$ & $\mathrm{C}_{13} \mathrm{H}_{9} \mathrm{I}_{2} \mathrm{~N}_{3} \mathrm{O}_{2}$ & $\mathrm{C}_{13} \mathrm{H}_{9} \mathrm{~F}_{2} \mathrm{~N}_{3} \mathrm{O}_{2}$ \\
\hline Formula weight & 354.59 & 354.59 & 493.03 & 277.23 \\
\hline Crystal system & Monoclinic & Monoclinic & Monoclinic & Monoclinic \\
\hline Space group & $P 2_{1} / c$ & $P 2_{1} / c$ & $P 2_{1} / c$ & $P 2_{1} / c$ \\
\hline $\mathrm{T}(\mathrm{K})$ & 298 & 368 & 298 & 298 \\
\hline$a(\AA)$ & $8.2475(9)$ & $8.651(2)$ & $8.2946(5)$ & $7.897(2)$ \\
\hline$b(\AA)$ & $10.8035(11)$ & $10.446(3)$ & $11.2673(6)$ & $10.396(2)$ \\
\hline$c(\AA)$ & 15.7971(15) & $15.680(4)$ & $16.2177(10)$ & 14.989(3) \\
\hline$\alpha(\operatorname{deg})$ & 90 & 90 & 90 & $90.00(3)$ \\
\hline$\beta(\operatorname{deg})$ & $96.098(3)$ & $94.116(11)$ & $97.993(2)$ & $92.27(3)$ \\
\hline$\gamma(\operatorname{deg})$ & 90 & 90 & 90 & $90.00(3)$ \\
\hline$V\left(\AA^{3}\right)$ & $1399.6(2)$ & $1413.3(7)$ & $1500.95(15)$ & $1229.6(5)$ \\
\hline$D_{\text {calcd }}\left(\mathrm{g} \mathrm{cm}^{-3}\right)$ & 1.683 & 1.667 & 2.182 & 1.498 \\
\hline$\mu\left(\mathrm{mm}^{-1}\right)$ & 3.131 & 3.101 & 4.194 & 0.124 \\
\hline$\theta$ range & 2.28 to 25.00 & 2.34 to 28.88 & 2.208 to 25.021 & 2.581 to 26.005 \\
\hline $\bar{Z} / \mathrm{Z}^{\prime}$ & $4 / 1$ & $4 / 1$ & $4 / 1$ & $4 / 1$ \\
\hline Range $h$ & -9 to 9 & -11 to 11 & -9 to 9 & -9 to 9 \\
\hline Range $k$ & -12 to 12 & -13 to 14 & -13 to 13 & -12 to 12 \\
\hline Range $l$ & -18 to 18 & -21 to 21 & -19 to 19 & -18 to 18 \\
\hline Reflections collected & 10084 & 23004 & 9640 & 30614 \\
\hline Total reflections & 2457 & 3582 & 2587 & 2336 \\
\hline
\end{tabular}




\begin{tabular}{|c|c|c|c|c|}
\hline Observed reflections & 2198 & 2117 & 2357 & 1807 \\
\hline$R_{1}[I>2 \sigma(I)]$ & 0.0354 & 0.0878 & 0.0272 & 0.0457 \\
\hline$w R_{2}$ (all) & 0.0990 & 0.2015 & 0.0698 & 0.1229 \\
\hline Goodness-of-fit & 1.077 & 1.143 & 1.111 & 1.096 \\
\hline X-ray diffractometer & Bruker D8 Quest & $\begin{array}{c}\text { Bruker D8 } \\
\text { Venture }\end{array}$ & Bruker D8 Quest & Bruker D8 Quest \\
\hline CCDC Numbers & 1548282 & 1548283 & 1548284 & 1548285 \\
\hline
\end{tabular}

Table S3: Stabilization energies of halogen bonds.

\begin{tabular}{|l|l|l|l|}
\hline Name of the Compound & Moiety & Stabilization Energy (kCal/mol) \\
\hline Compound-A Form I & \\
\hline Compound-A Form II & \\
\hline
\end{tabular}



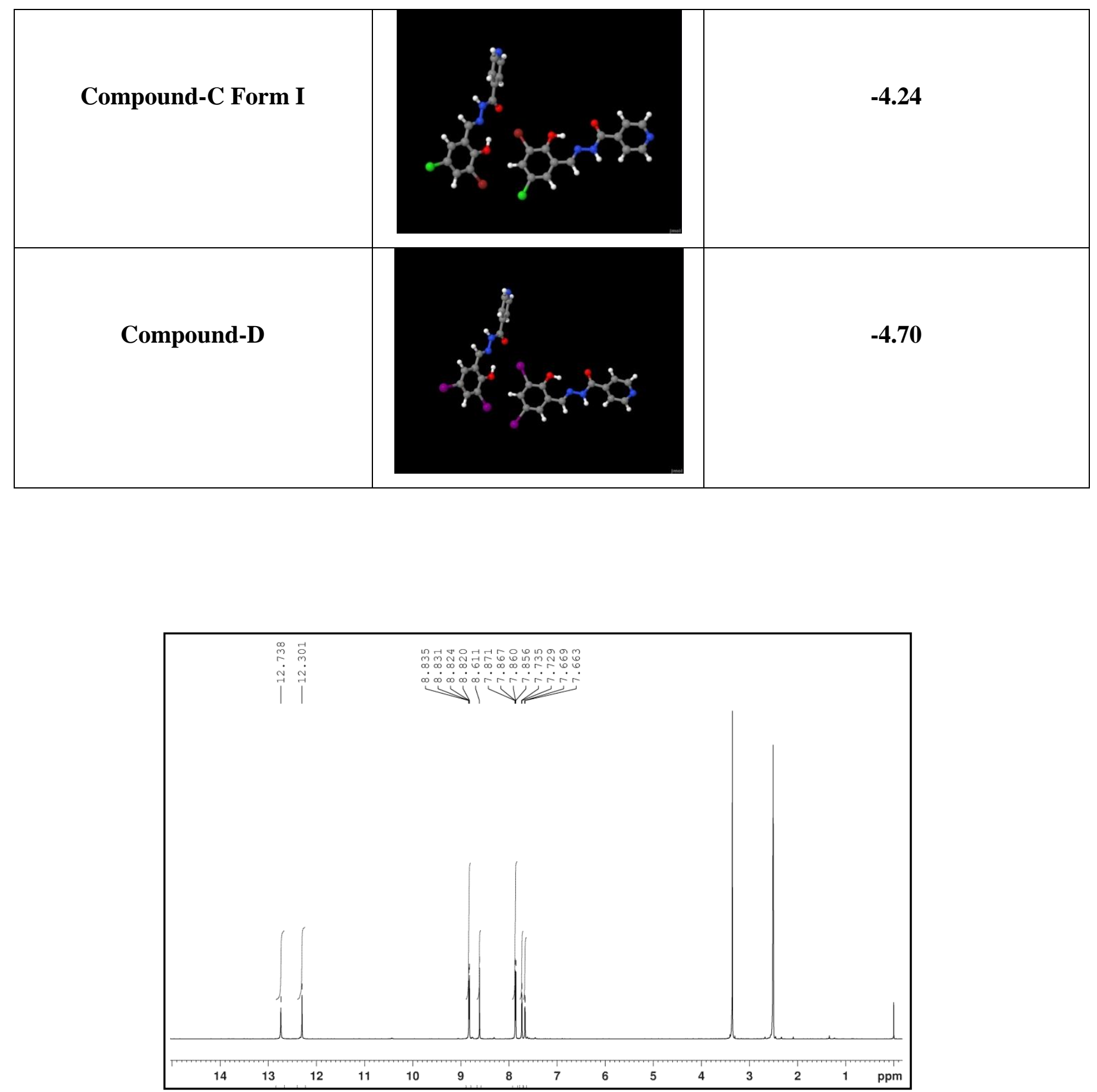

Figure S1a: Compound-A ${ }^{1} \mathrm{H}-\mathrm{NMR}$. 


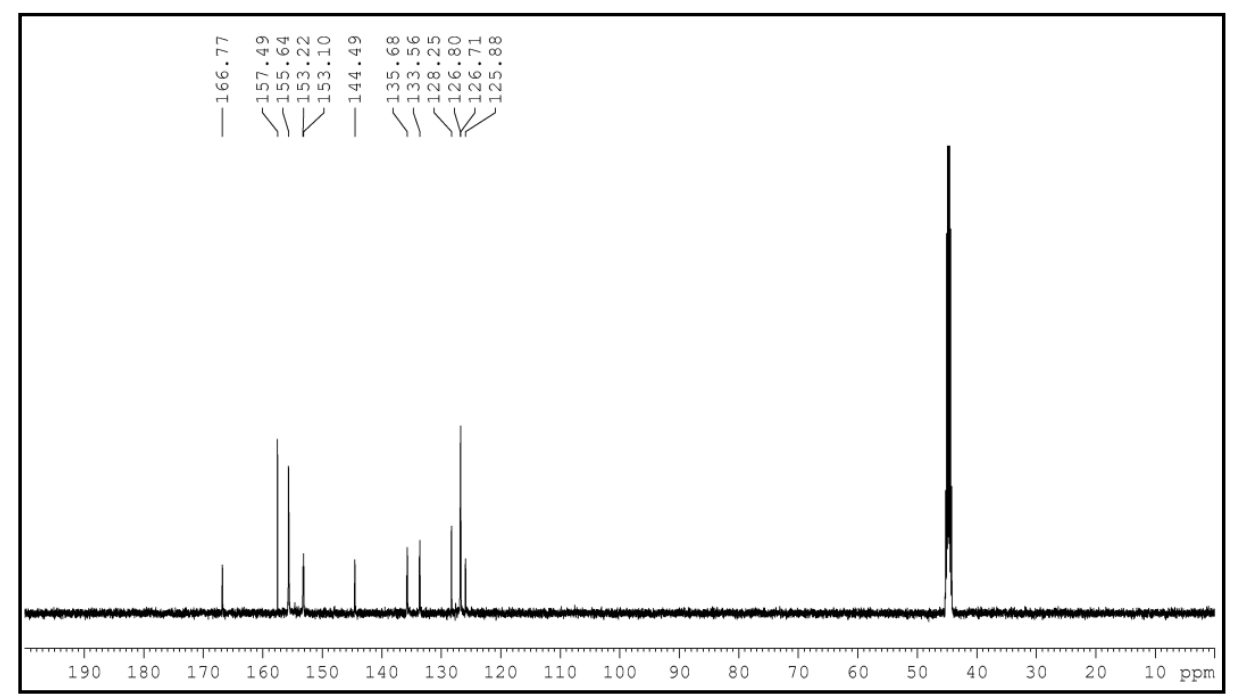

Figure S1b: Compound-A ${ }^{13} \mathrm{C}-\mathrm{NMR}$.

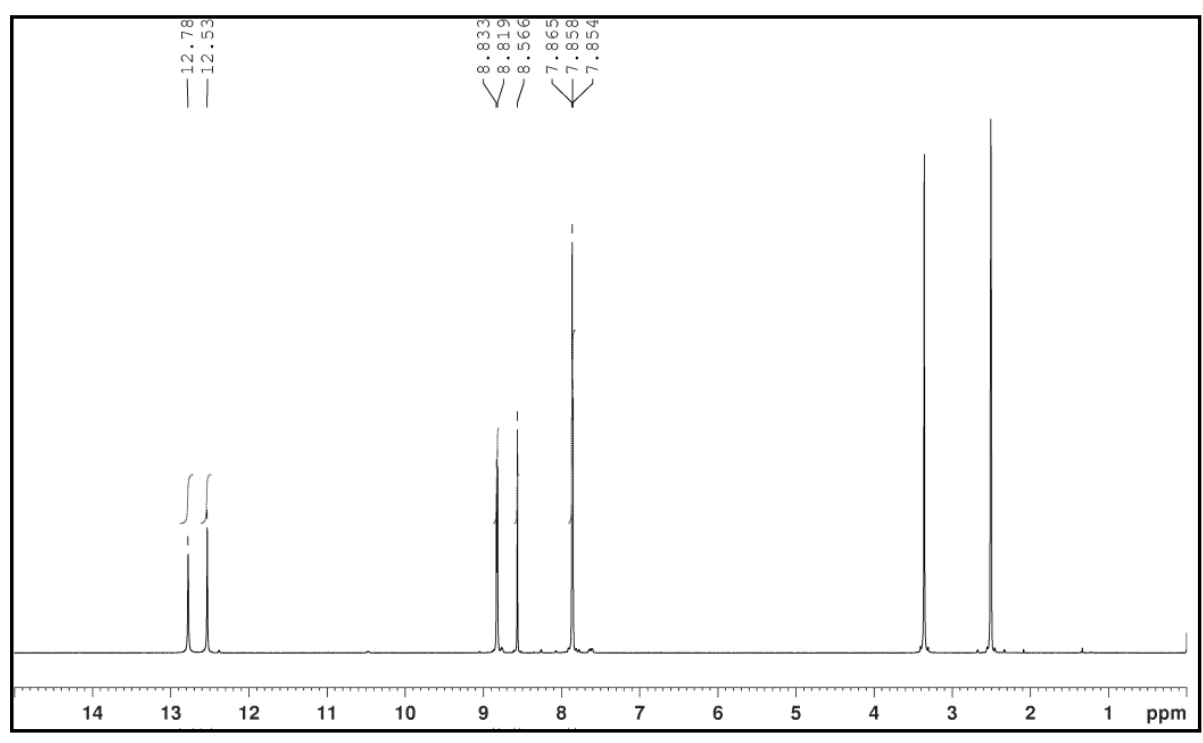

Figure S1c: Compound-B ${ }^{1} \mathrm{H}-\mathrm{NMR}$. 


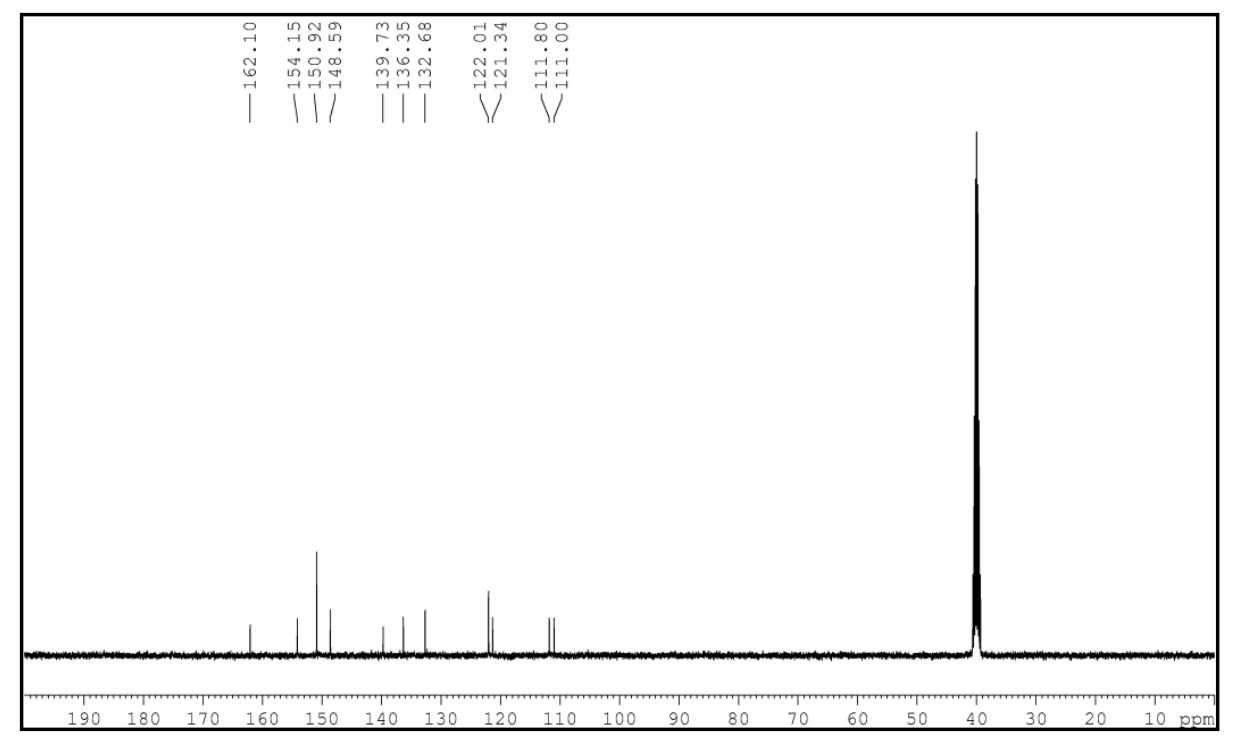

Figure S1d: Compound-B ${ }^{13} \mathrm{C}-\mathrm{NMR}$.

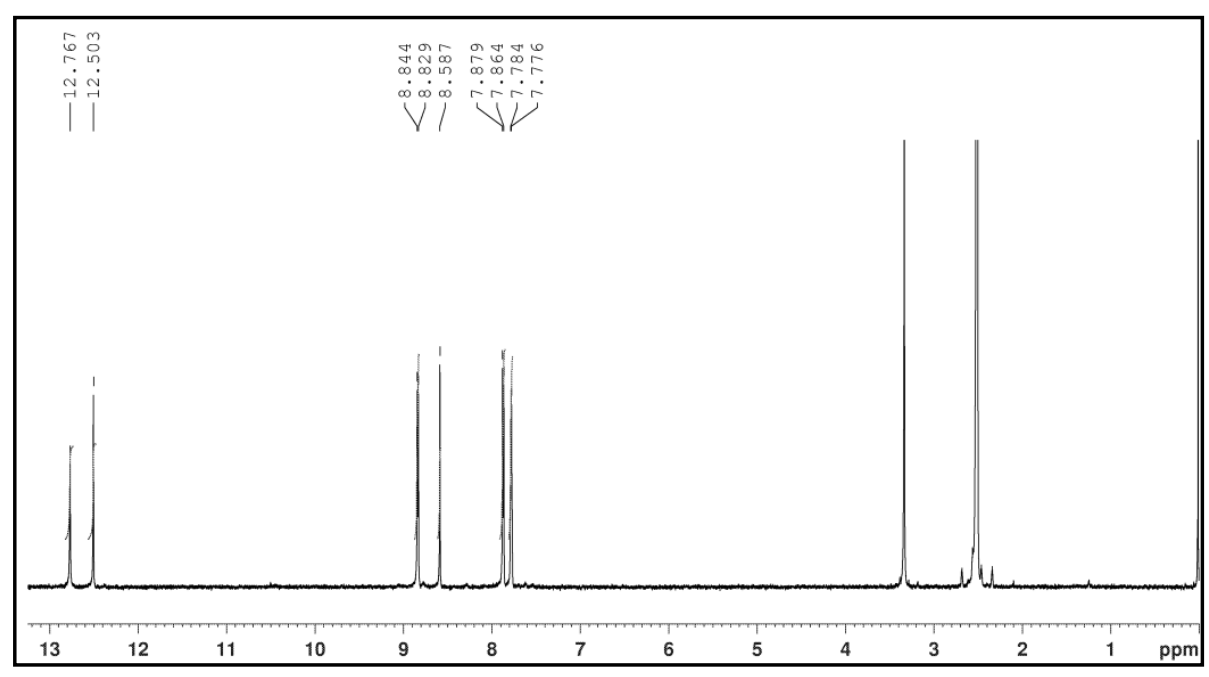

Figure S1e: Compound-C ${ }^{1} \mathrm{H}-\mathrm{NMR}$. 


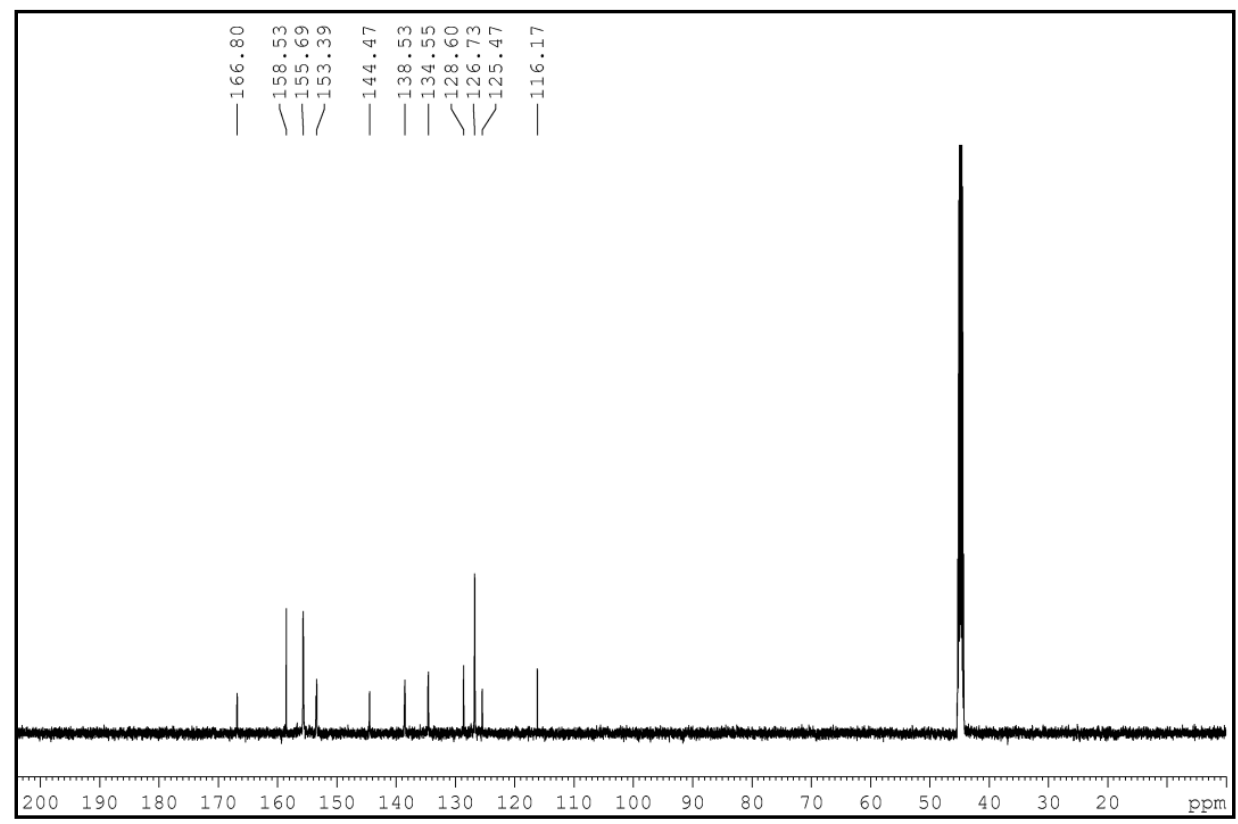

Figure S1f: Compound-C ${ }^{13} \mathrm{C}-\mathrm{NMR}$.

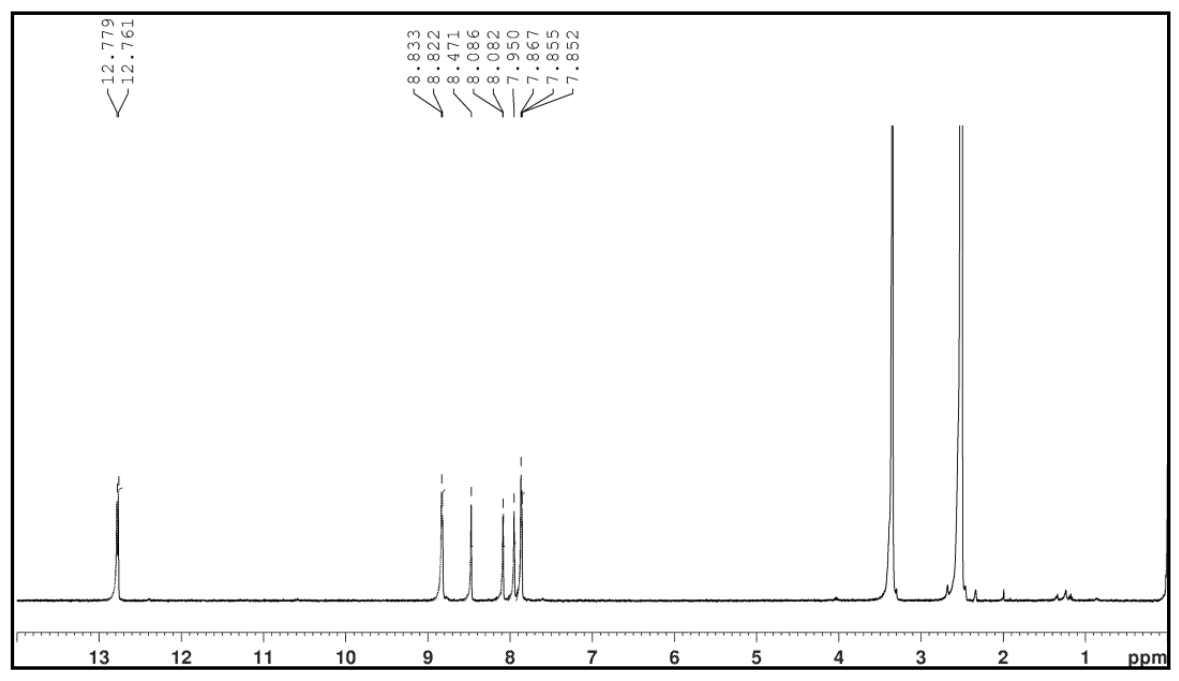

Figure S1g: Compound-D ${ }^{1} \mathrm{H}-\mathrm{NMR}$. 


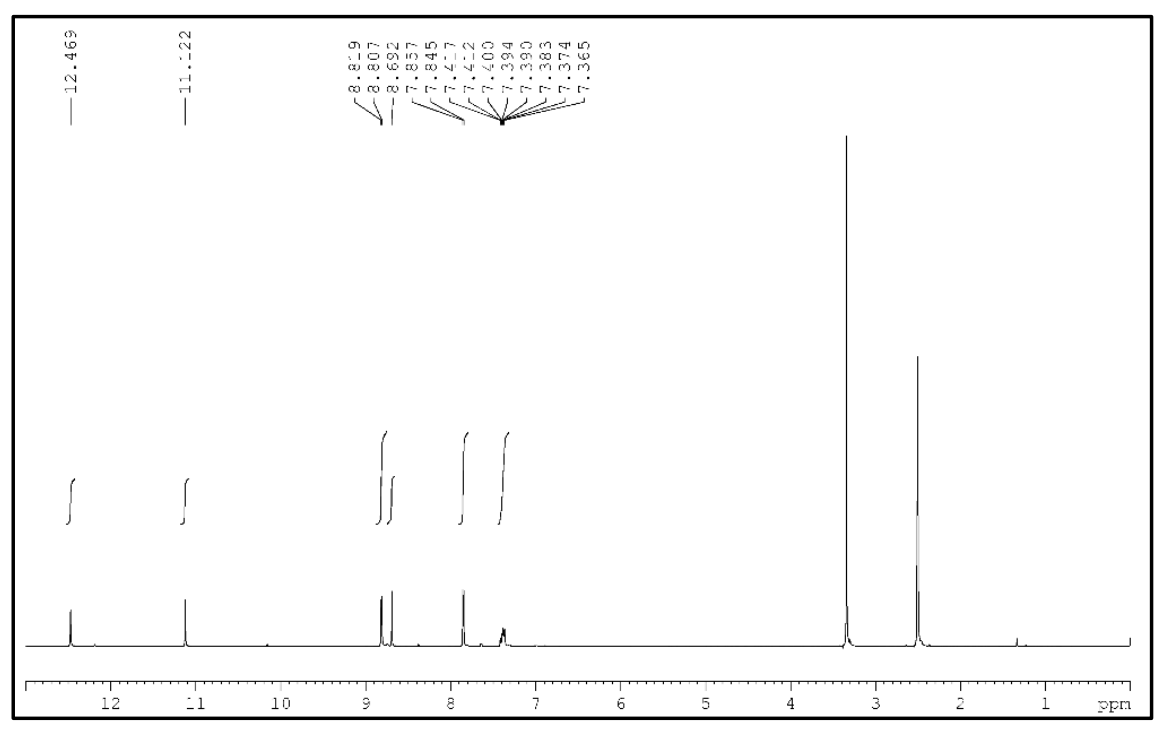

Figure S1h: Compound-E ${ }^{1} \mathrm{H}-\mathrm{NMR}$.

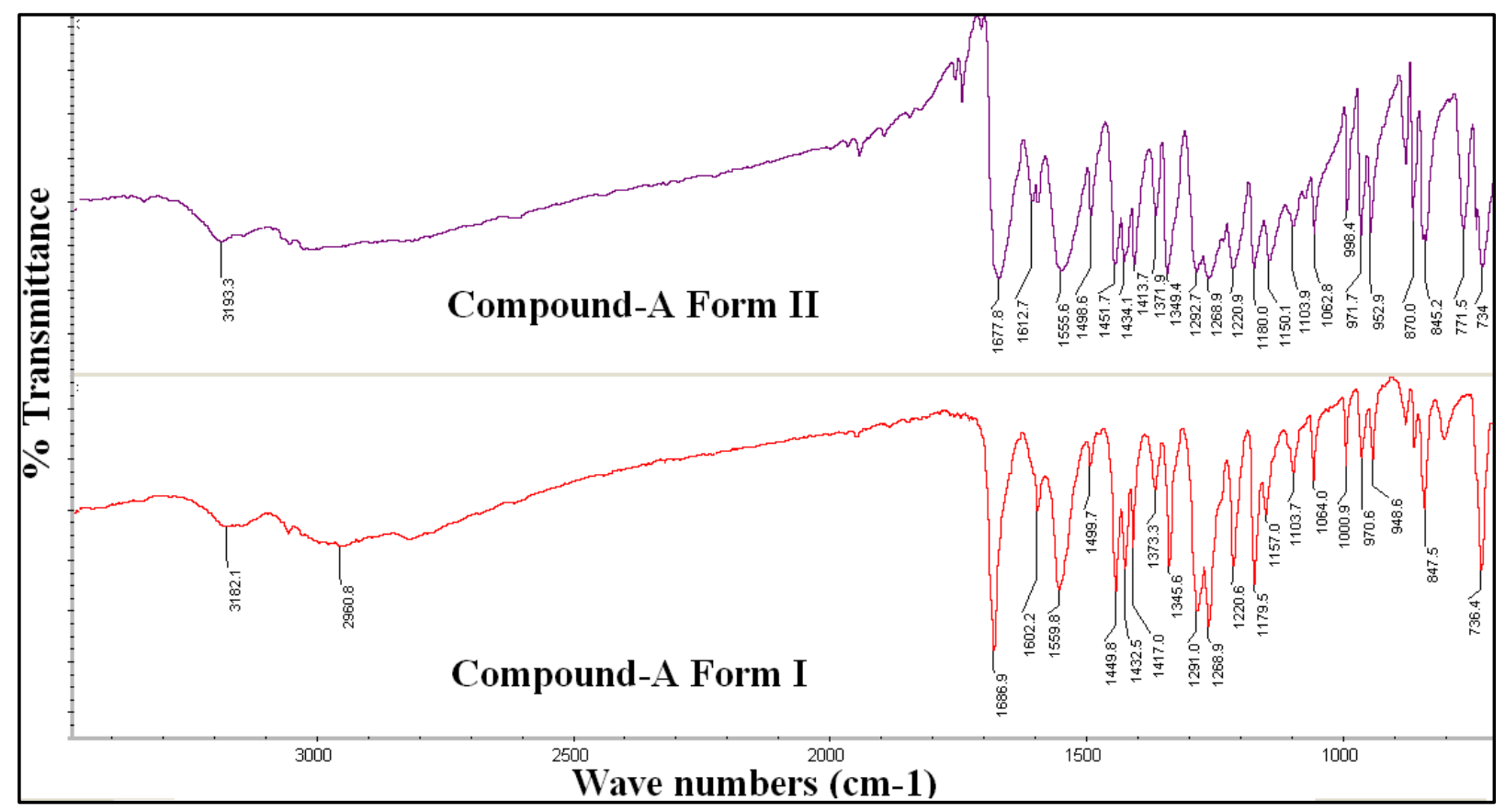

Figure S2a: FT-IR comparison for Form I and Form II (Compound-A) 


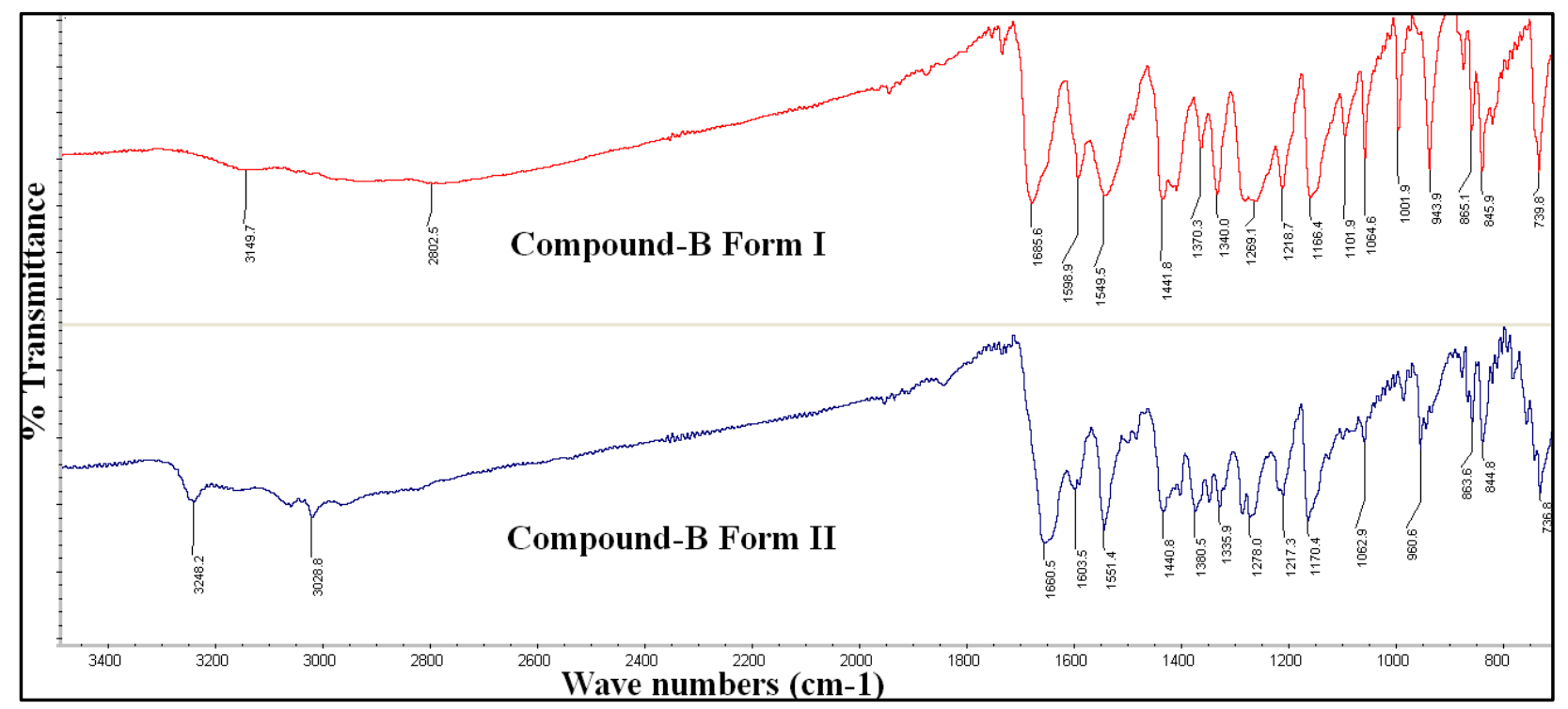

Figure S2b: FT-IR comparison for Form I and Form II (Compound-B)

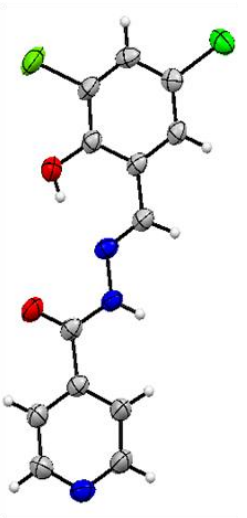

A

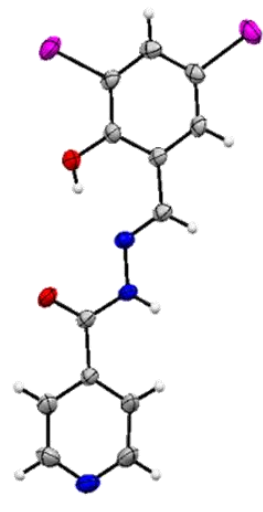

B

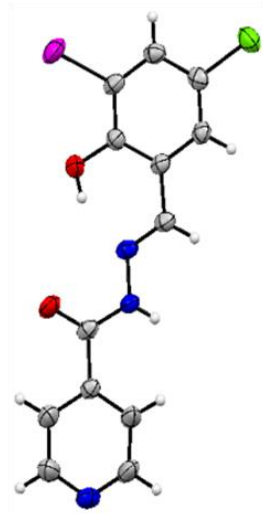

C

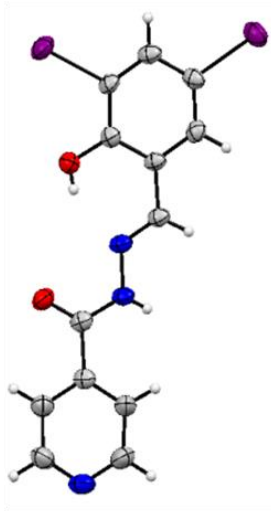

D

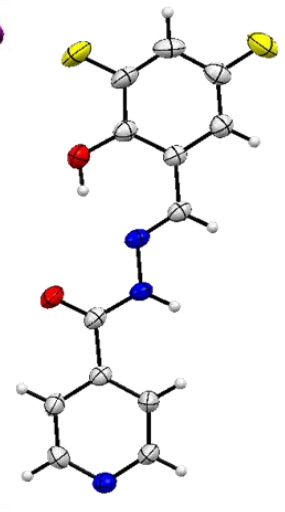

$\mathbf{E}$

$\mathrm{A}=(\mathrm{E})-\mathrm{N}$-(3,5-dichloro-2-hydroxybenzylidene)isonicotinohydrazide

$\mathrm{B}=(\mathrm{E})-\mathrm{N}$ '-(3,5-dibromo-2-hydroxybenzylidene)isonicotinohydrazide

$\mathrm{C}=(\mathrm{E})-\mathrm{N}$-(3-bromo-5-chloro-2-hydroxybenzylidene)isonicotinohydrazide

$\mathrm{D}=(\mathrm{E})-\mathrm{N}^{\prime}$-(3,5-diiodo-2-hydroxybenzylidene)isonicotinohydrazide

$\mathrm{E}=(\mathrm{E})-\mathrm{N}$ '-(3,5-difluoro-2-hydroxybenzylidene)isonicotinohydrazide

Figure S3: ORTEP diagram of salinazids structures. 


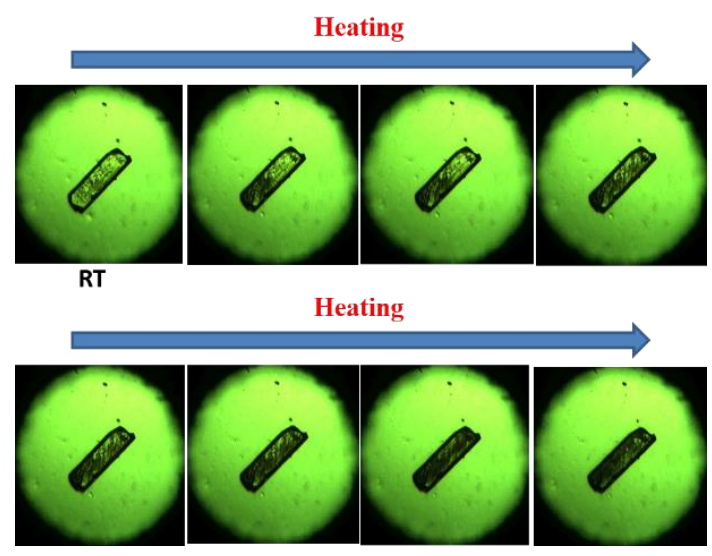

Figure S4: Hot stage microscope images during heating of Compound-B thermosalient crystals.

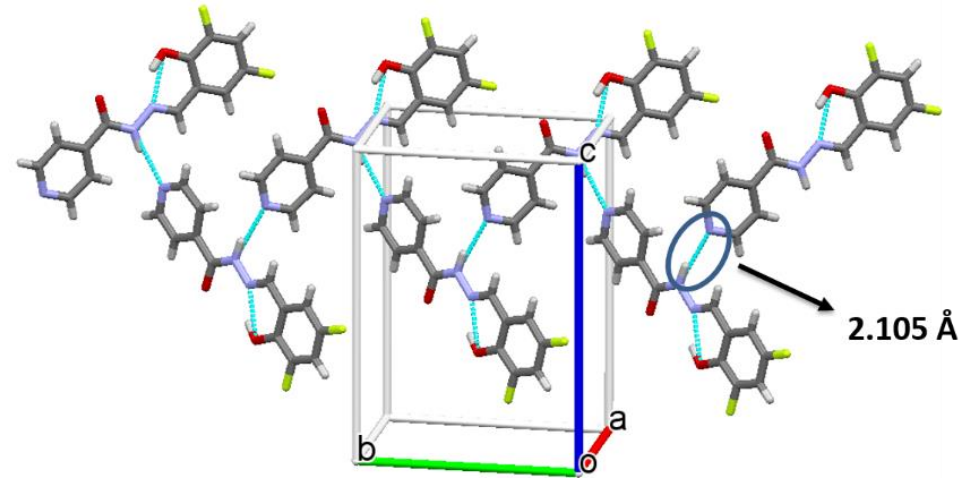

Figure S5: Chain extending through N-H...N H bond interactions (Compound-E).

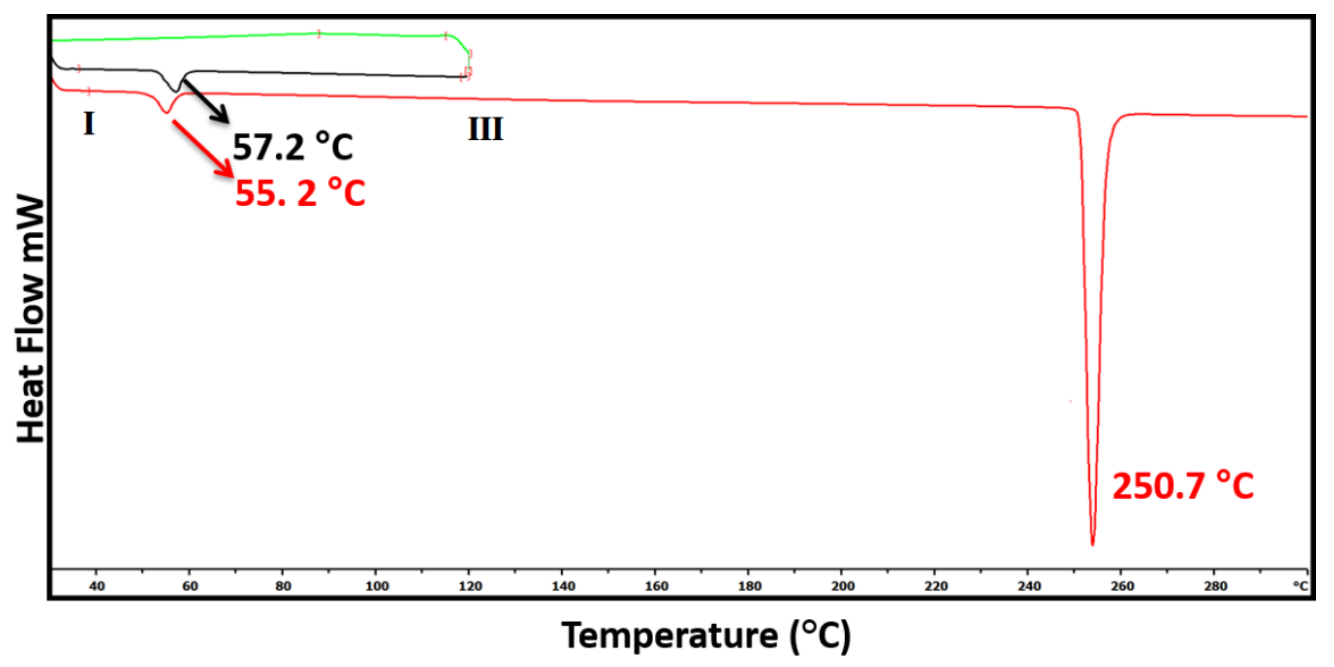

Figure S6a: DSC thermogram of Compound-A Form I. 


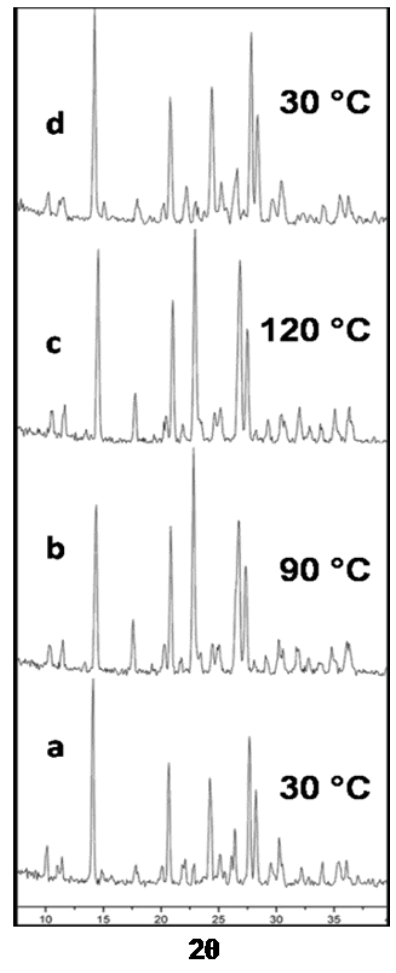

a

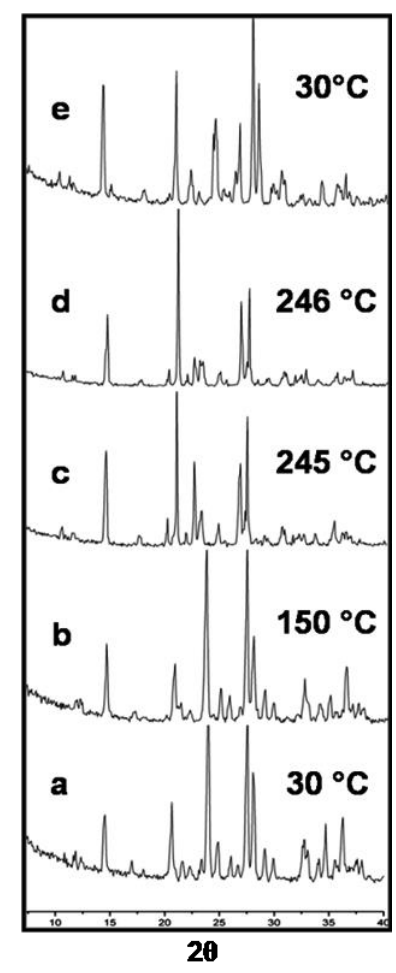

b

Figure S6b: VT-PXRD for Compound-A Form I (a) and II (b).

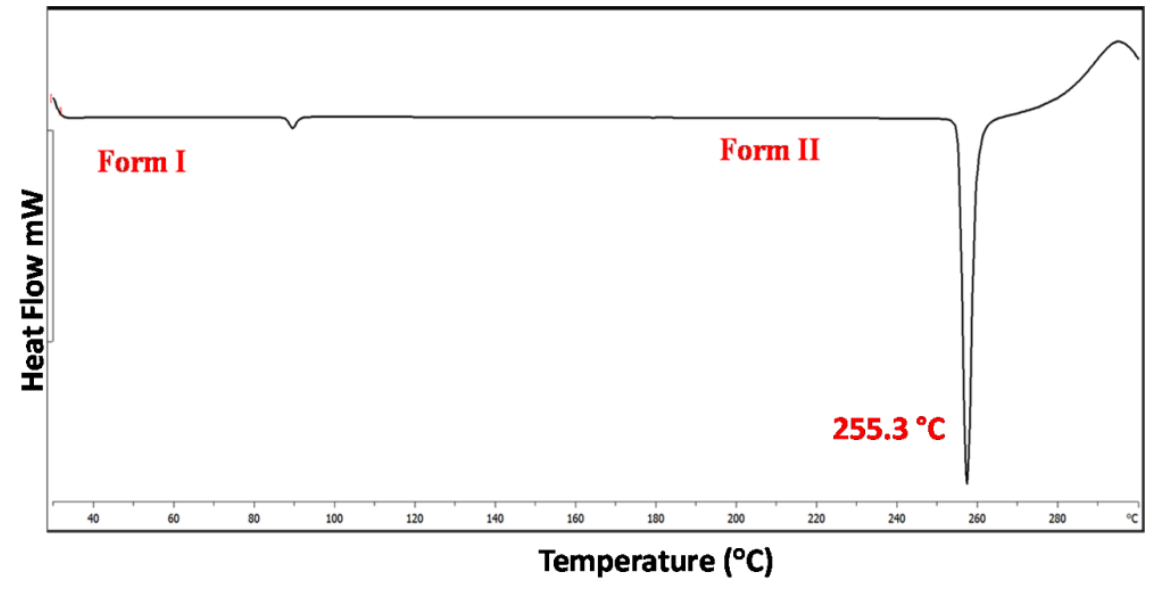

Figure S7a: DSC thermogram of Compound-C. 


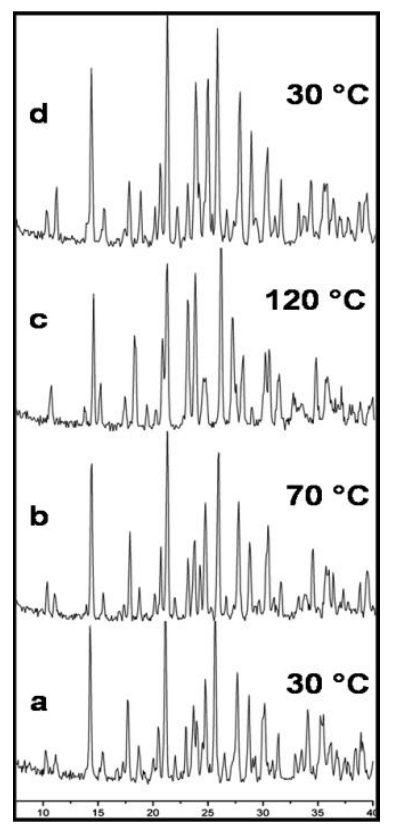

Figure S7b: VT-PXRD for Compound-C Form I (label a) which converts to Form II at $120{ }^{\circ} \mathrm{C}$ (label c).

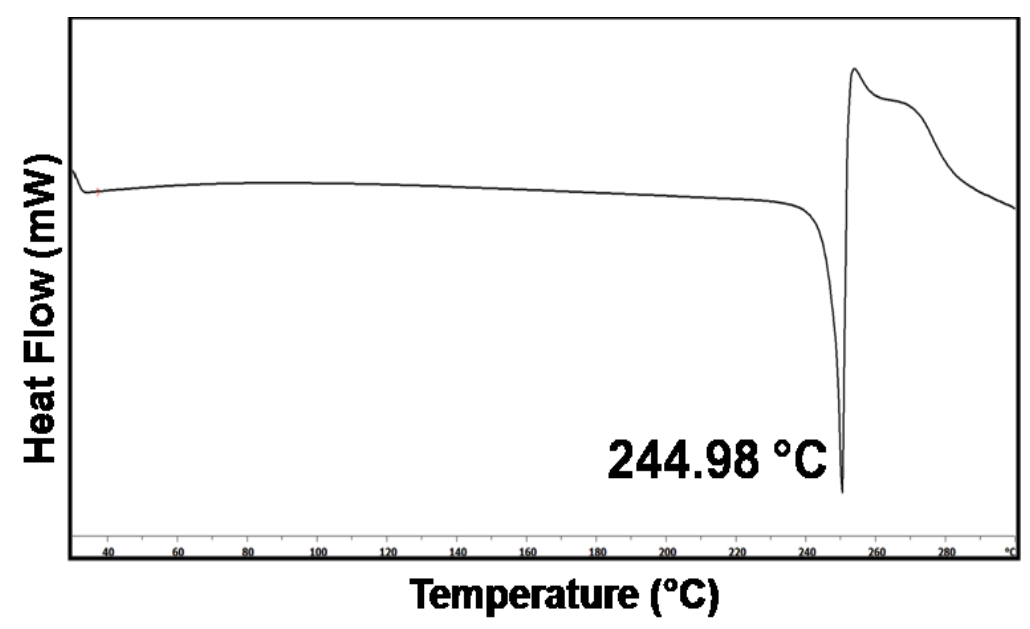

Figure S8: DSC thermogram of Compound-D. 


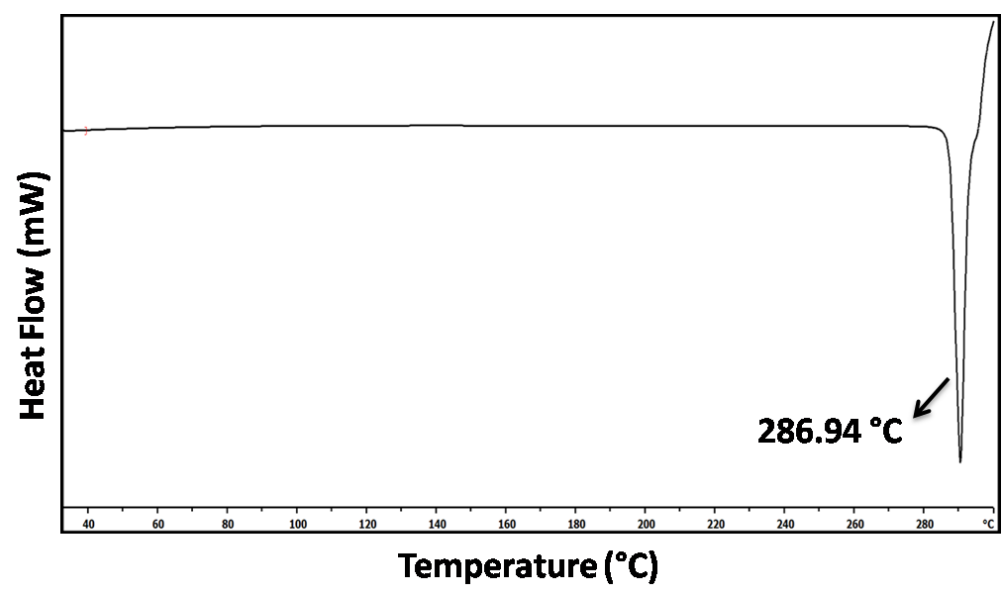

Figure S9: DSC thermogram of Compound-E. 


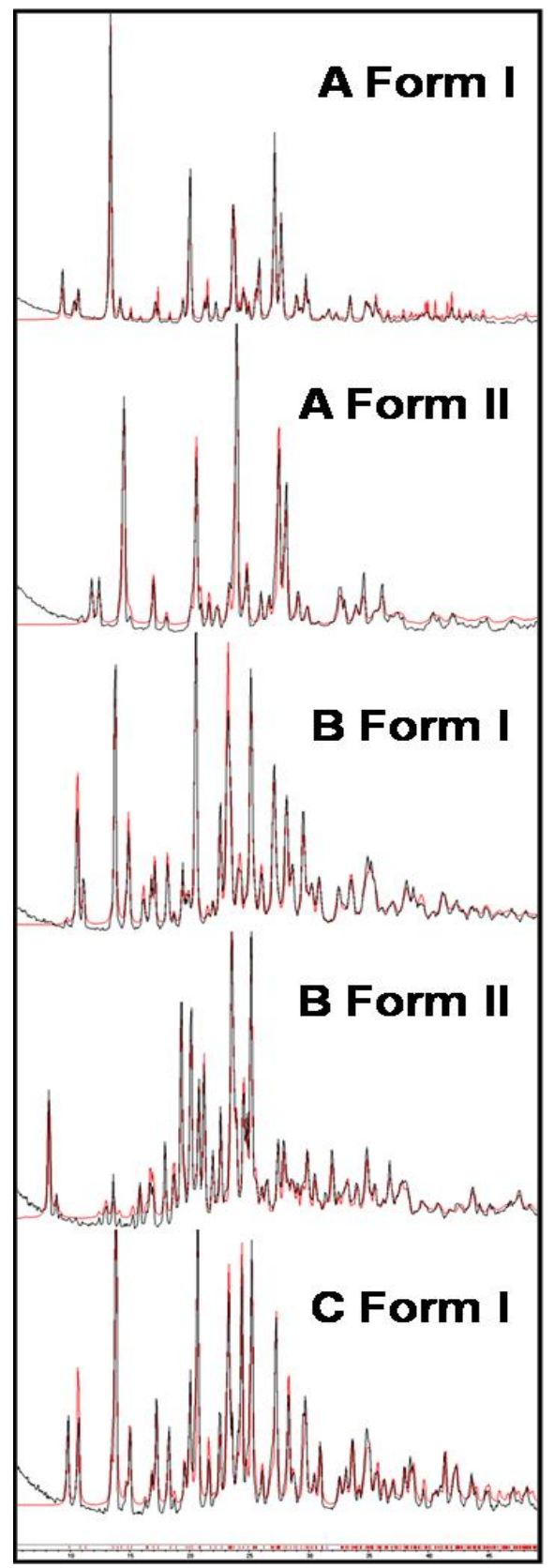

Figure S10: Overlay of experimental PXRD pattern with calculated lines from the X-ray structure. 


\section{XPac Analysis:}

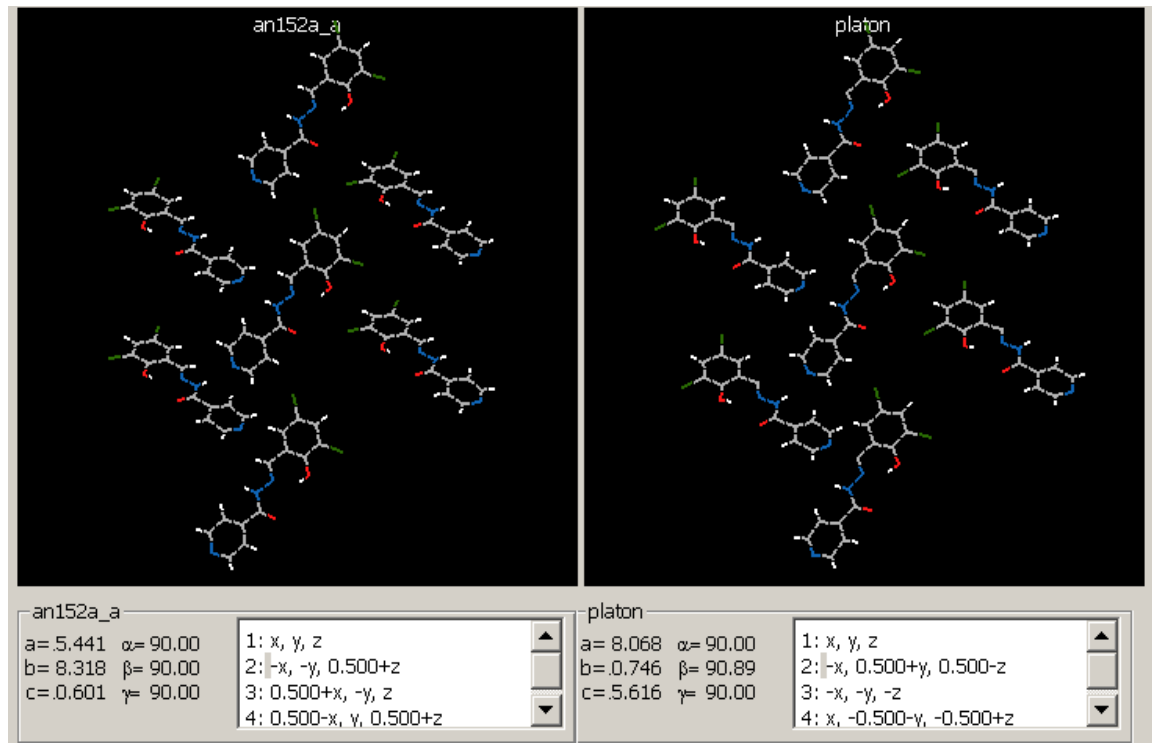

(a)

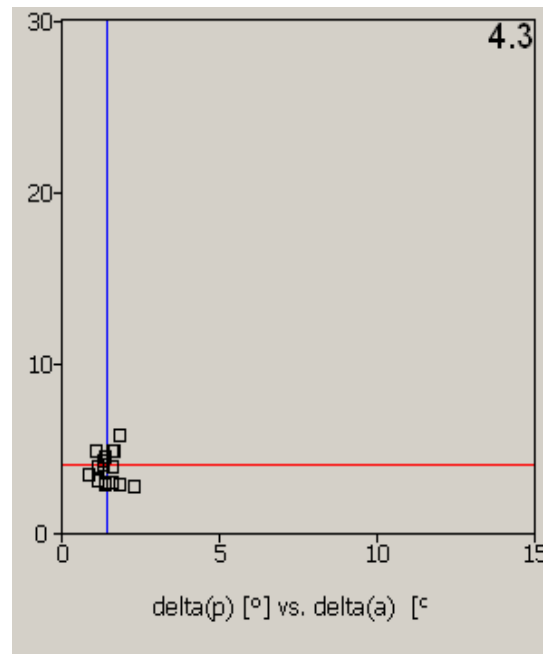

(b)

Figure S11a: (a) XPac analysis of compound-A Form I and II. (b) The interplanar angular deviation ( $\delta \mathrm{p}, \mathrm{x}$-axis) vs. angular deviation ( $\delta \mathrm{a}, \mathrm{y}$-axis) plot $\left(\mathrm{in}^{\circ}\right)$ indicates a dissimilarity index of 4.3 due to the 3D isostructurality. 


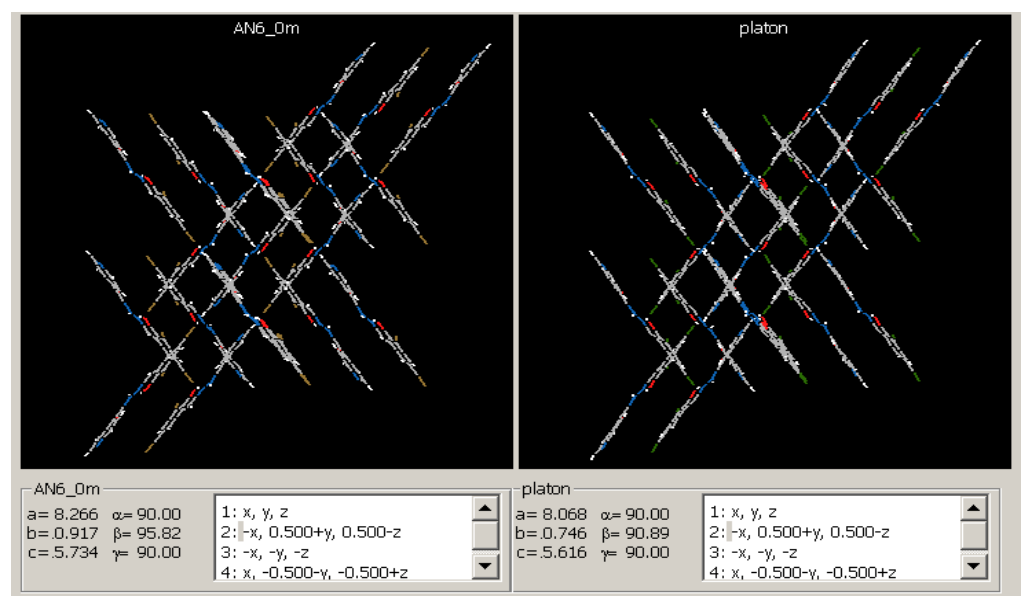

(a)

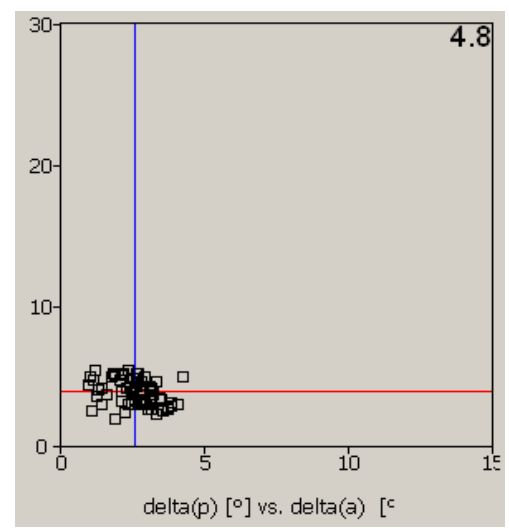

(b)

Figure S11b: (a) XPac analysis of compound-A and B. (b) The interplanar angular deviation ( $\delta p$, $\mathrm{x}$-axis) vs. angular deviation ( $\delta \mathrm{a}, \mathrm{y}$-axis) plot (in ${ }^{\circ}$ ) indicates a dissimilarity index of 4.8 due to the $3 \mathrm{D}$ isostructurality. 


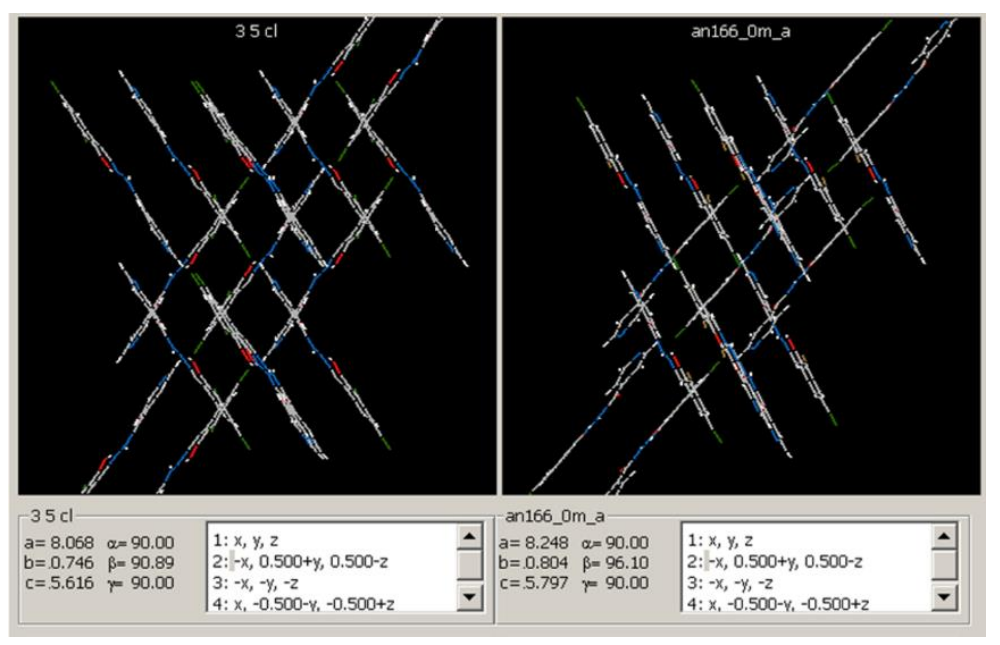

(a)

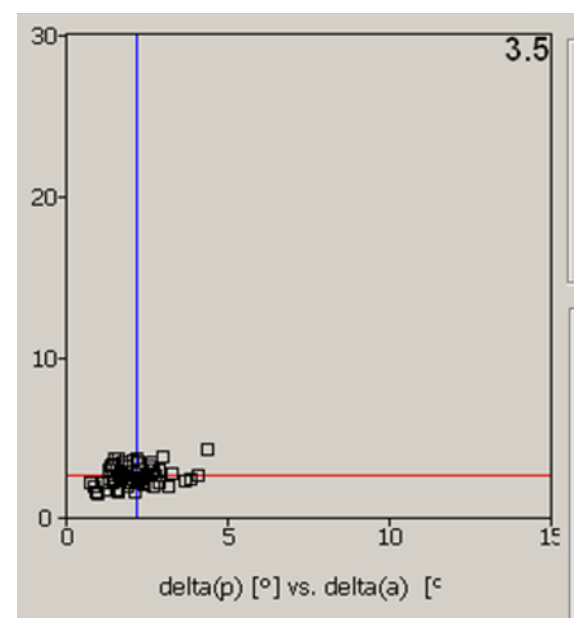

(b)

Figure S11c: (a) XPac analysis of compound-A and C. (b) The interplanar angular deviation ( $\delta p$, $\mathrm{x}$-axis) vs. angular deviation ( $\mathrm{Ca}, \mathrm{y}$-axis) plot (in ${ }^{\circ}$ ) indicates a dissimilarity index of 3.5 due to the $3 \mathrm{D}$ isostructurality. 


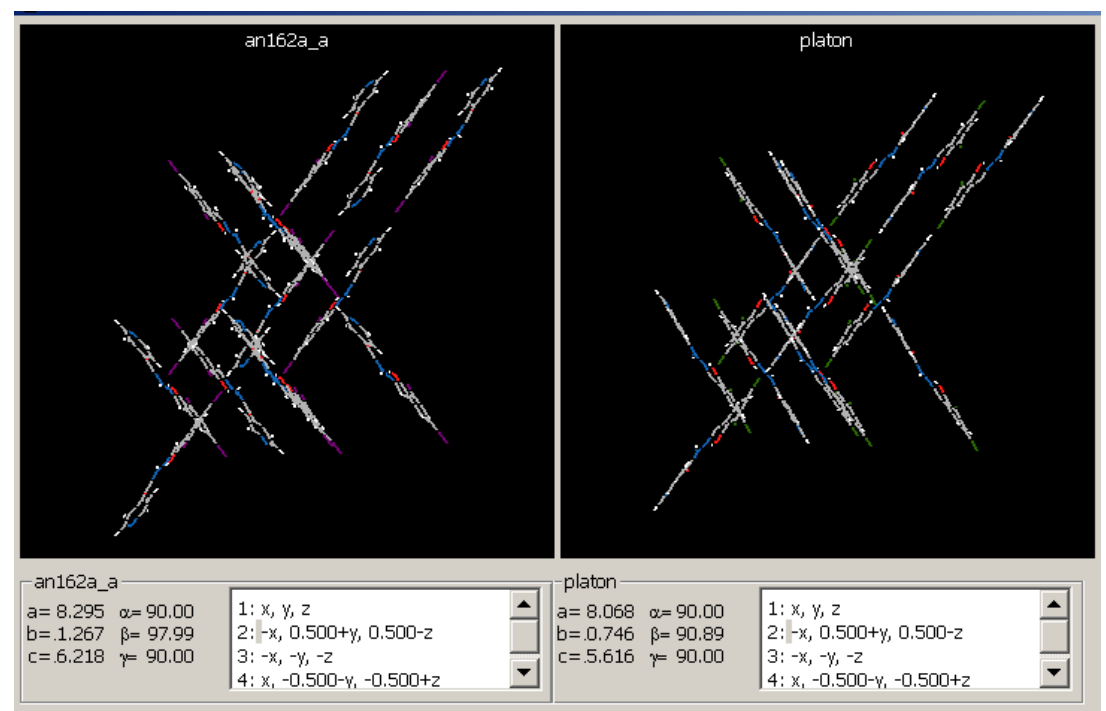

(a)

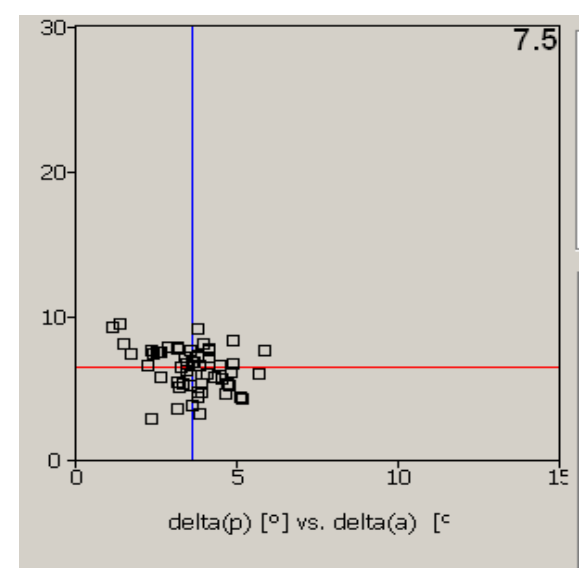

(b)

Figure S11d: (a) XPac analysis of compound-A and D. (b) The interplanar angular deviation ( $\delta p$, $\mathrm{x}$-axis) vs. angular deviation ( $\delta \mathrm{a}, \mathrm{y}$-axis) plot (in ${ }^{\circ}$ ) indicates a dissimilarity index of 7.5 due to the $3 \mathrm{D}$ isostructurality. 


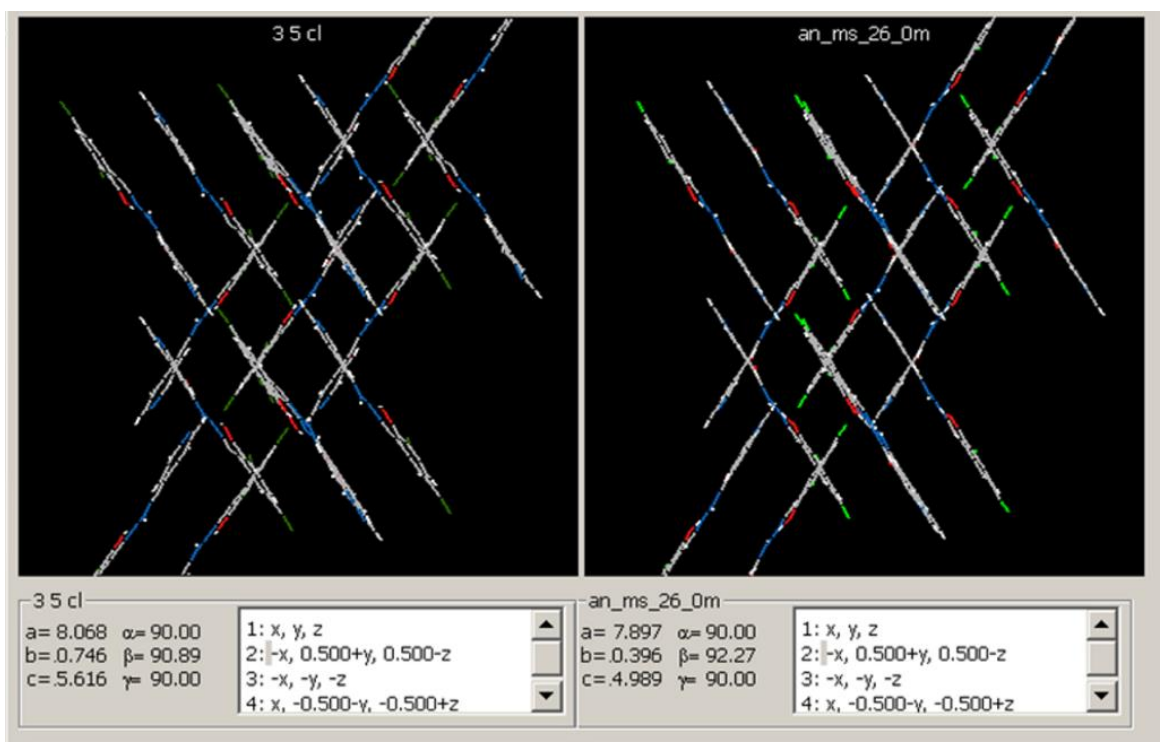

(a)

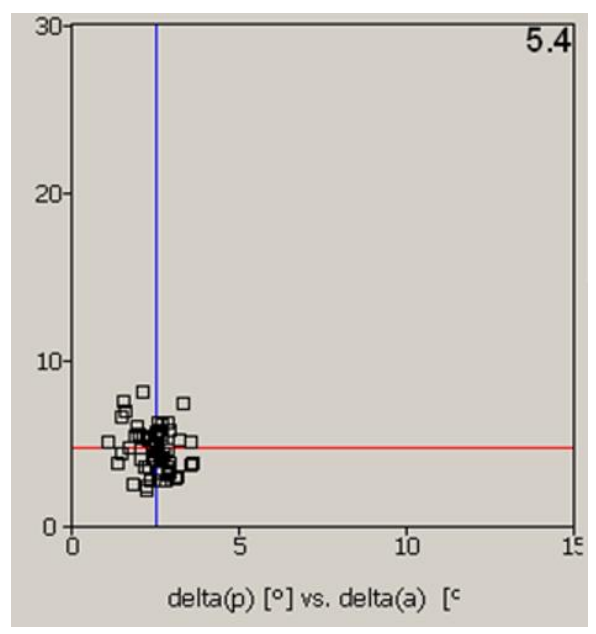

(b)

Figure S11e: (a) XPac analysis of compound-A and E. (b) The interplanar angular deviation ( $\delta p$, $\mathrm{x}$-axis) vs. angular deviation ( $\delta \mathrm{a}, \mathrm{y}$-axis) plot (in ${ }^{\circ}$ ) indicates a dissimilarity index of 5.4 due to the $3 \mathrm{D}$ isostructurality. 
Hirshfeld Surface Analysis:

\section{Compound-A Form I:}
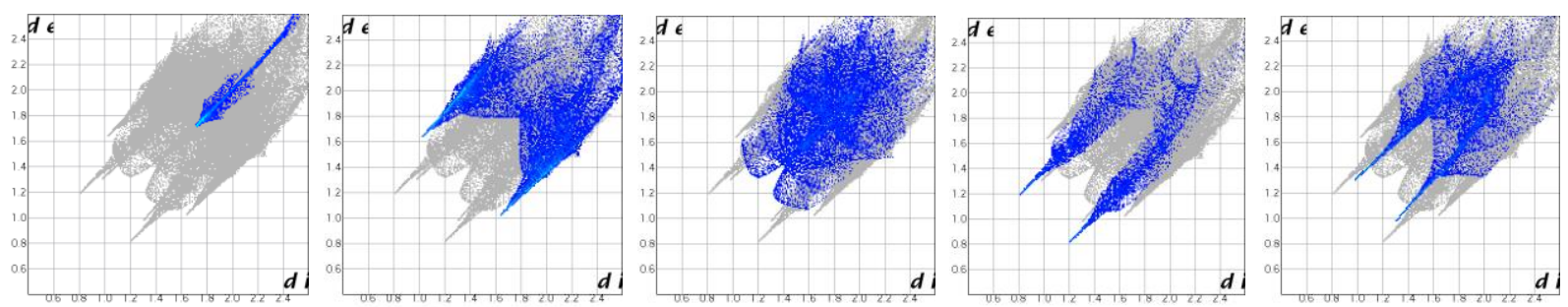

Cl...Cl 3.0\%

Cl...H 21.9\%

H...H $19.1 \%$

N...H 7.6\%

O...H 13.2\%

\section{Compound-A Form II:}
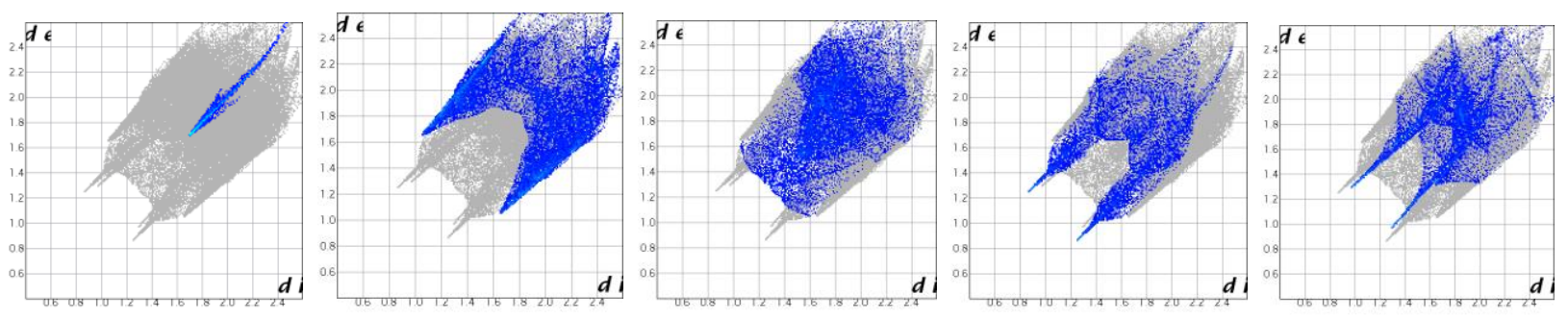

Cl...Cl 2.8\%

Cl...H 21.7\%

N...H $10.2 \%$

O...H $12.6 \%$

\section{Compound-A Form III:}
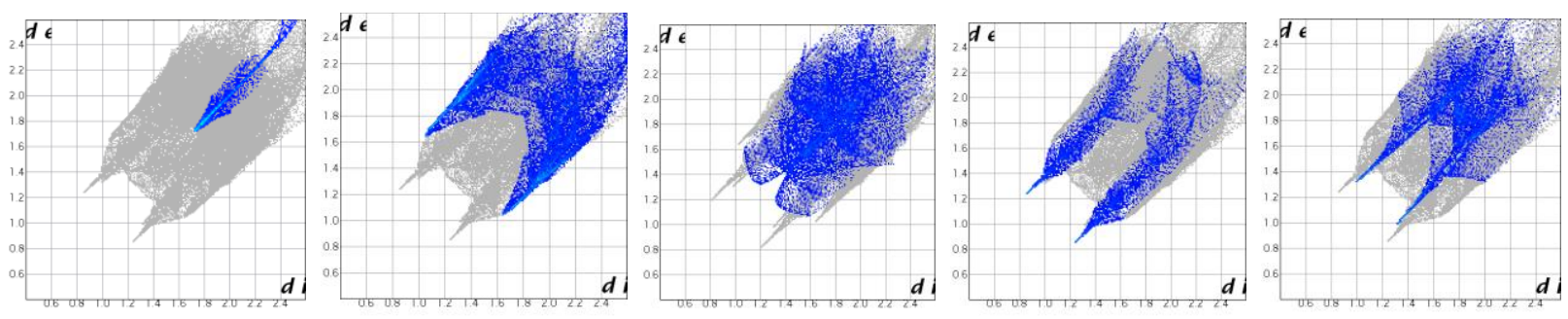

Cl...Cl 3.9\%
Cl...H 21.5\%
N...H 8.4\%

O...H 13.6\% 


\section{Compound-B Form I:}
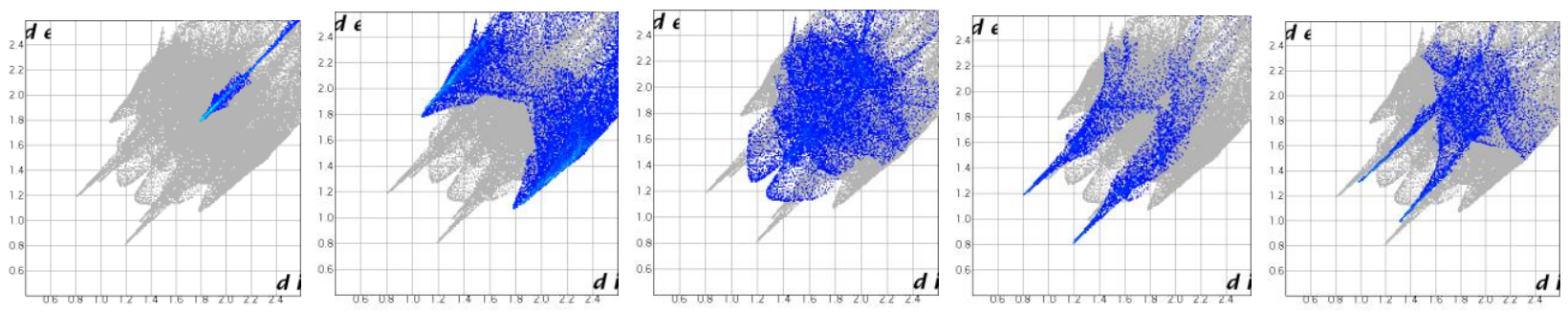

Br...Br 3.2\%

Br...H 23.2\%

H...H $18.6 \%$

N...H 7.4\%

O...H $9.8 \%$

\section{Compound-B Form II:}
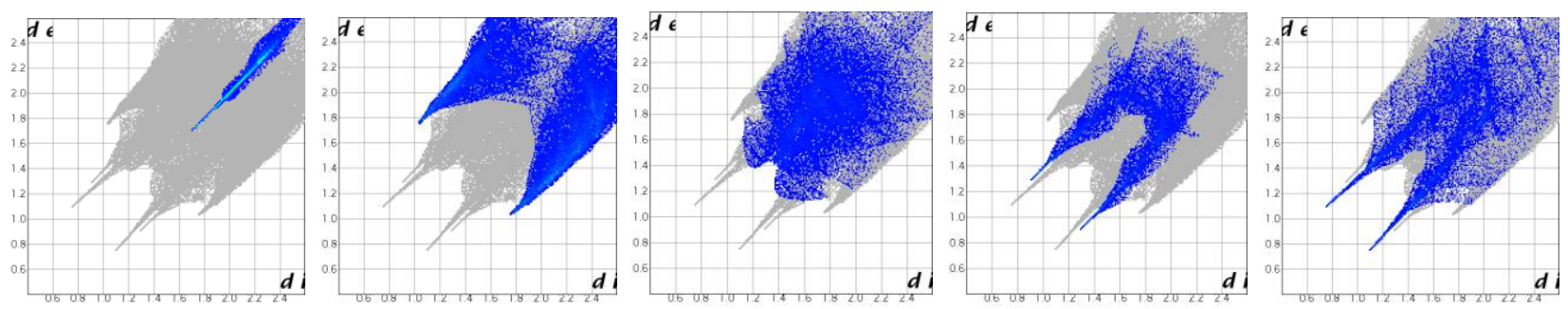

Br...Br 5.9\% Br...H 25.8\%

H...H $15.8 \%$

N...H 8.1\%

O...H 11.5\%

\section{Compound-C Form I:}
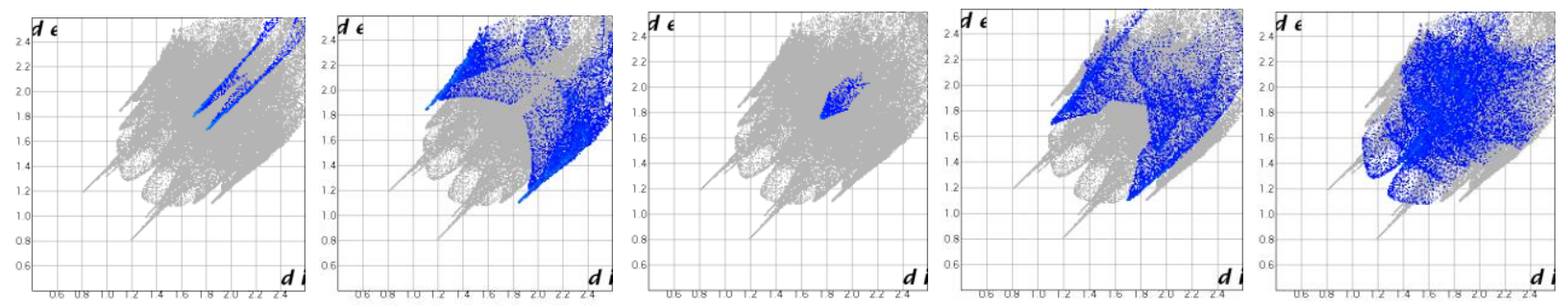

Br...Cl 2.6\%

Br...H 12.9\%

Cl...Cl 0.7\%

Cl...H 9.7\%

H...H 20.3\% 

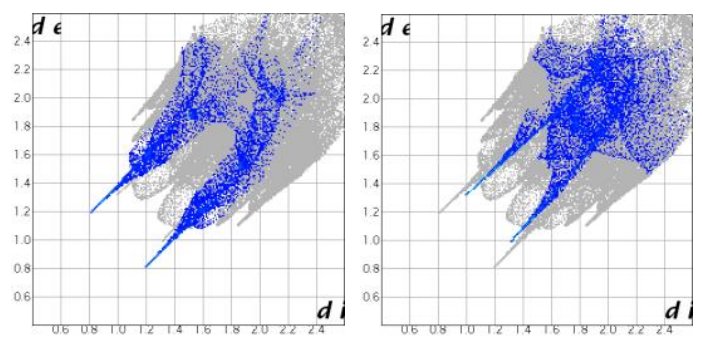

N...H $7.7 \%$

О...H 10.6\%

\section{Compound-C Form II:}
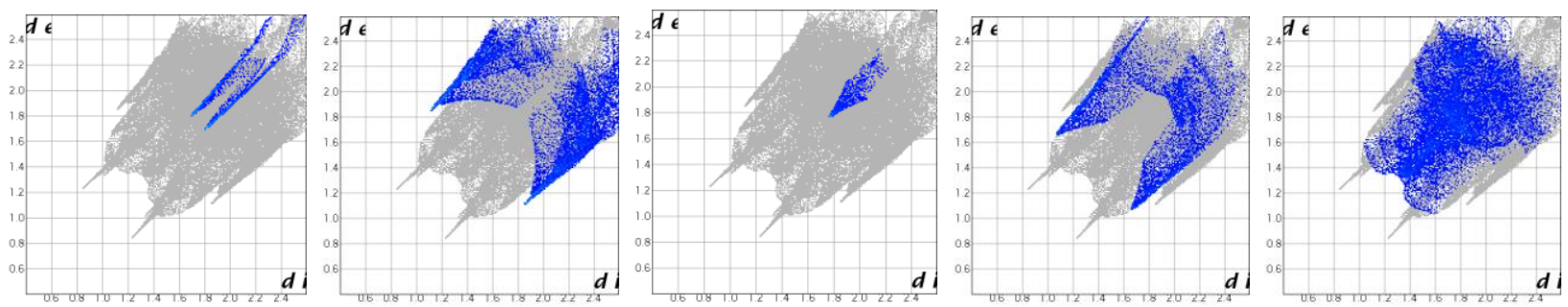

Br...Cl 2.8\%

Br...H 13.2\%

Cl...Cl 1.1\%

Cl...H 8.4\%

H...H 20.5\%
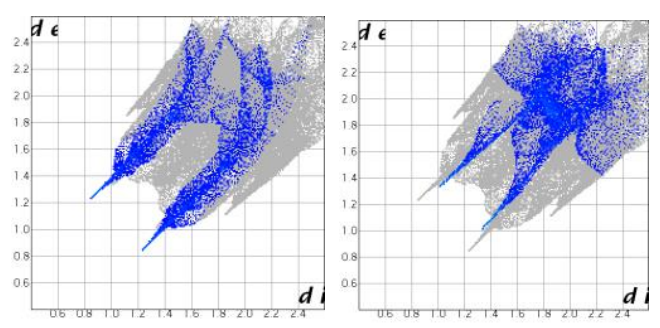

N...H $8.4 \%$

О...H 12.2\%

\section{Compound-D:}
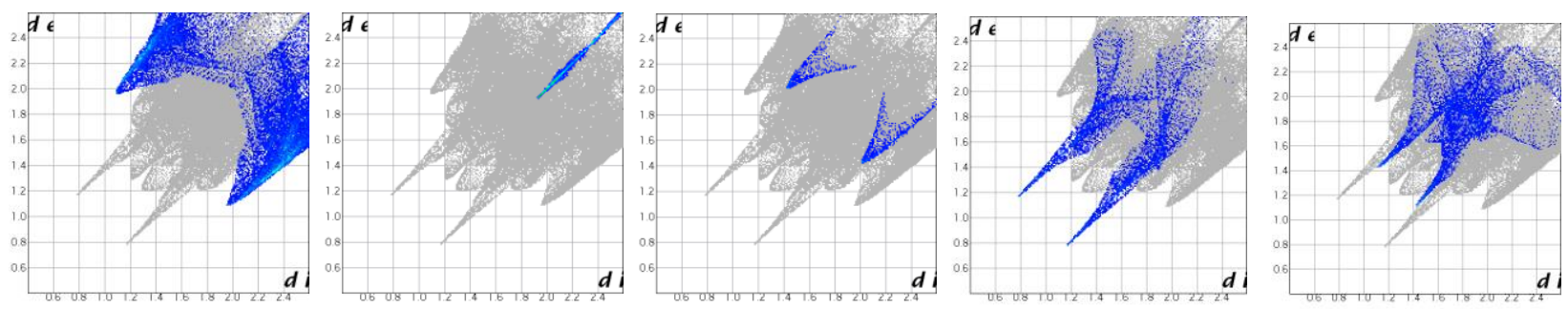

I...H $21.3 \%$
I...O $1.5 \%$
N...H 7.1\%

О...H 7.9\% 
Figure S12: Hirshfeld Surface analysis for Compound A-D.

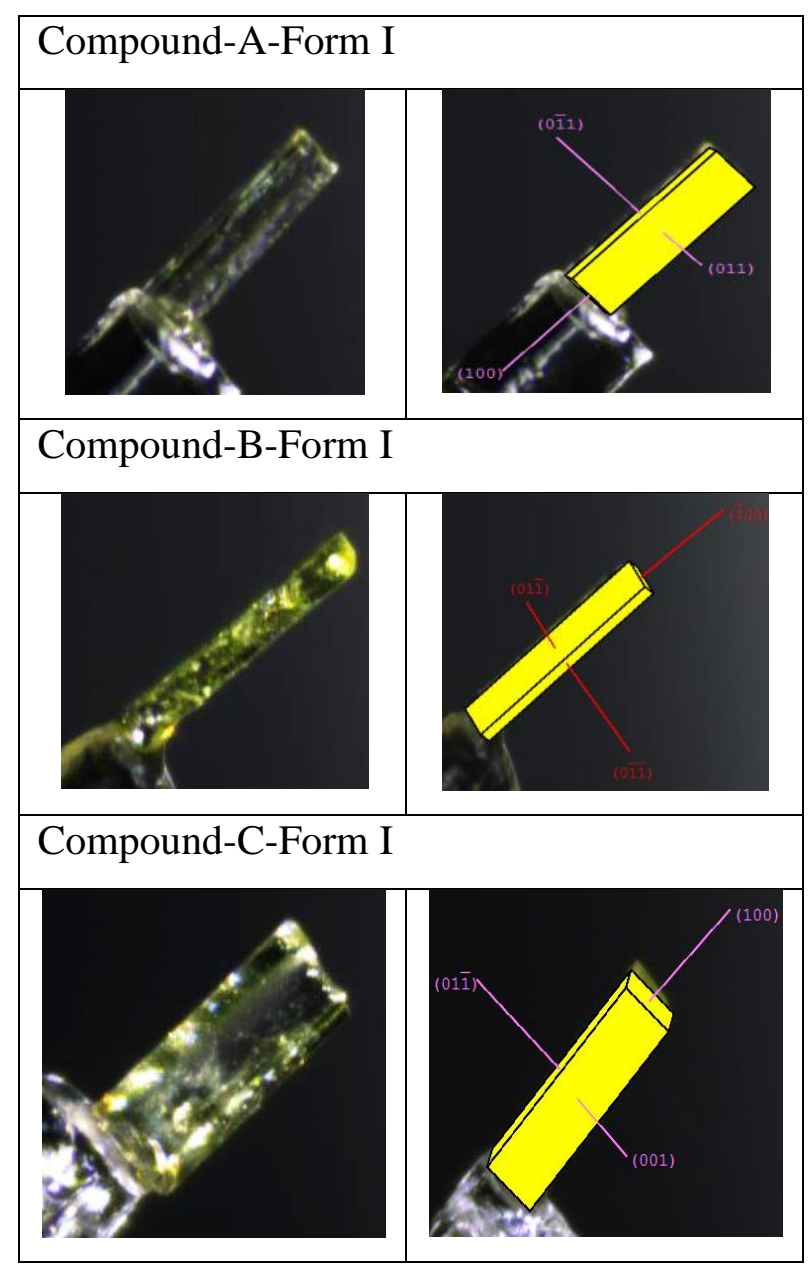

Figure S13: Morphology and faces of crystals. 